\title{
The QCD confinement transition: hadron formation
}

\author{
Francesco Becattini \\ Università di Firenze and INFN Sezione di Firenze, \\ Via G. Sansone 1, I-50019, Sesto Fiorentino (Firenze), Italy \\ becattini@fi.infn.it \\ and \\ R. Fries \\ Cyclotron Institute, Texas A \& M University, \\ College Station, TX 77843, USA \\ and RIKEN/BNL Research Center, Brookhaven National Laboratory, Upton, NY \\ 11973, USA \\ rjfries@comp.tamu.edu
}




\section{Introduction}

A major phenomenon that the theory of strong interactions, quantum chromodynamics (QCD), should account for is confinement: quarks and gluons are not observable particles. In fact, every physical process involving strong interactions at high energy results in the formation of hadrons, in which quarks and gluons are confined on a distance scale of $\mathcal{O}(1)$ fm. While, up to now, there is no formal proof that QCD implies confinement, there are many indications, both from perturbative and from lattice numerical studies, that this is likely the case. Perturbative QCD is applicable to scattering processes of quarks and gluons involving large momentum transfer ( $\gg 1 \mathrm{GeV}$ ) because the strong coupling constant $\alpha_{S}$ is small enough to allow a series expansion. However, this is no longer possible at a scale of $1 \mathrm{GeV}$ or below, where the perturbative expansion is meaningless, and where confinement and hadronization, the process of hadron formation, takes place. Thus, hadronization is not yet calculable from QCD first principles and one has to resort to phenomenological models. While this may seem an inconvenient limitation, still much can be learned from these models about QCD in the confinement regime. Indeed, if they are able to effectively describe the essential features of the actual physical process, they give us relevant information about the characteristics of the fundamental theory.

In this chapter, we will review two of these models, that have found widespread use in relativistic heavy ion collisions. The first is a model with a rather long history that has recently been revived by its successes in the description of hadronic multiplicities, the statistical model. This model is applicable to hadronization in elementary collisions as well as heavy ion collisions. This is in fact its main strength, i.e. it captures a universal feature in the hadronization process.

The second model is the quark recombination or coalescence model which extends the concept of single parton fragmentation function, which has been used in elementary collisions since the '70s. Its recent success comes from observations specific to relativistic heavy ion collisions.

We will start by reviewing the foundations and the main results of the statistical model in Sect. 2 and of the quark recombination model in Sect. 3. In Sect. 4 we will compare the two models and discuss further perspectives in the understanding of the hadronization phenomenon.

\section{The Statistical Hadronization Model}

The idea of applying statistical concepts to the problem of multi-particle production in high energy collisions dates back to a work of Fermi [1] in 1950, who assumed that particles originated from an excited region evenly occupying all available phase space states. This was one of Fermi's favorite ideas and soon led to an intense effort in trying to work out the predictions of inclusive particle rates calculating, analytically and numerically, the involved multidimensional phase-space integrals. When it became clear that the (quasi) isotropic particle emission in the center-of-mass frame predicted by 
Fermi's model was ruled out by the data, an amendment was put forward by Hagedorn [2] in the '60s, who postulated the existence of two hadron emitting sources flying apart longitudinally in the center-of-mass frame of a pp collision. Thereby, one could explain the striking difference between spectra in transverse and longitudinal momentum. Hagedorn was also able to explain the almost universal slope of $p_{T}$ spectra in his renowned statistical bootstrap model, assuming that resonances are made of hadrons and resonances in turn.

After QCD turned up, many phenomenological models of strong interactions were no longer pursued and the statistical model was no exception. The resurgence of interest in these ideas came about when it was argued that a completely equilibrated hadron gas would be a clear signature of the formation of a transient Quark-Gluon Plasma (QGP) in heavy ion collisions at high energy. While it has been indeed confirmed that an (almost) fully equilibrated hadron gas has been produced [3] in those collisions, the interest in this model was also revived by the unexpected observation that it is able to accurately reproduce particle multiplicities in elementary collisions [4]. Naively, one did not expect a statistical approach to work in an environment where the number of particles is $\mathcal{O}(10)$ because it was a belief of many that a hadronic thermalization process would take a long time if driven by hadronic collisions. Apparently this is not the case and one of the burning questions, which is still waiting a generally accepted answer, is why a supposedly non-thermal system exhibits a striking thermal behavior.

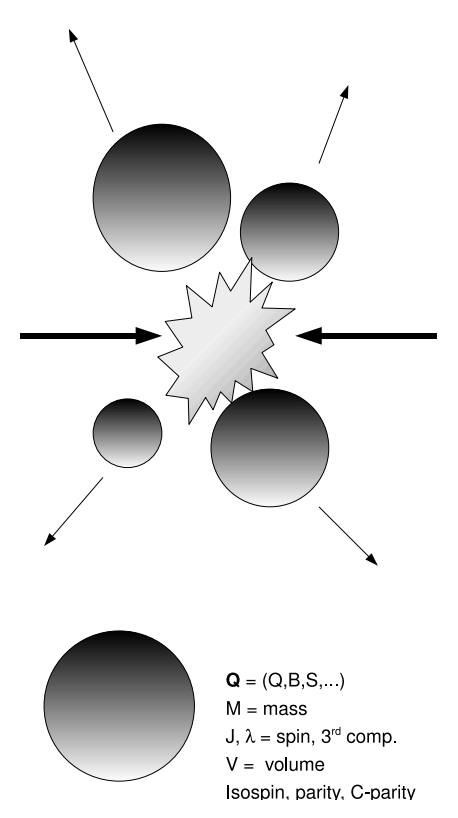

Figure 1: High energy collisions are assumed to give rise to multiple clusters at the hadronization stage [top]. Each cluster [bottom] is a colorless extended massive object endowed with abelian charges (electric, strange, baryonic etc.), intrinsic angular momentum and other quantum numbers such as parity, $C$-parity and isospin. 
Before we address this interesting issue, it is appropriate to provide a rigorous formulation of the model in a modern form, which is necessarily different from Fermi's original model due to the tremendous improvement in our knowledge of strong interactions phenomenology. The Statistical Hadronization Model (SHM) must be considered as an effective model describing the process of hadron formation in high energy collisions at energy (or distance) scales where perturbative QCD is no longer applicable. A high energy collision is thought of as a complex dynamical process, governed by QCD, which eventually gives rise to the formation of extended massive colorless objects defined as clusters or fireballs (see Fig. 1). While the multiplicity, masses, momenta and charges of these objects are determined by this complex dynamical process, the SHM postulates that hadrons are formed from the decay of each cluster in a purely statistical fashion, that is:

Every multihadronic state localized within the cluster and compatible with conservation laws is equally likely.

This is the urprinzip of the SHM. The assumption of the eventual formation of massive colorless clusters is common for many hadronization models (e.g. the cluster model implemented in the Monte-Carlo code HERWIG [5]) based on the property of color preconfinement [6] exhibited by perturbative QCD. The distinctive feature of the SHM is that clusters have a finite spacial size. This aspect of clusters as a relativistic massive extended objects coincides with that of a bag in the MIT bag model [7]. Indeed, the SHM can be considered as an effective model to calculate bag decays.

The requirement of finite spacial extension is crucial. If the SHM is to be an effective representation of the QCD-driven dynamical hadronization process, this characteristic must be ultimately related to the QCD fundamental scale $\Lambda_{\mathrm{QCD}}$. As we will see, the universal soft scale shows up in the approximately constant energy density at hadronization; in other words, the volume of clusters is in a constant ratio with their mass when hadronization takes place. It is also worth stressing here that there is clear, independent evidence of the finite size of hadronic sources in high energy collisions. Quantum interference effects in the production of identical particles, the so-called Bose-Einstein correlations or Hanbury Brown-Twiss second-order interference, is by now a firmly established phenomenon. This effect would simply be impossible without a finite volume.

\section{$2.1 \quad$ Localized States}

The basic postulate of the Statistical Hadronization Model asserts that every localized multihadronic state which is contained within a cluster and is compatible with conservation laws is equally likely. The word localized, implying a finite spacial size, plays a crucial role, as we have emphasized. Thus, before getting to the heart of the SHM formalism, it is necessary to pause and clarify the distinction between localized and asymptotic states.

Such a difference is not an issue when the volume is sufficiently larger than the Compton wavelength of hadrons and it is disregarded in most applications where clusters 
supposedly meet this requirement (e.g. heavy ion collisions); yet, it is an important point at a fundamental level. Although in thermodynamics the focus is on the limit of infinite volumes, we must start from a finite volume and localized systems to introduce concepts like energy density, temperature etc. Furthermore, in the hadronic world, finite size effects must be diligently taken into account when the volume is comparable to the (third power of) the pion's Compton wavelength, $\sim 1.4 \mathrm{fm}$.

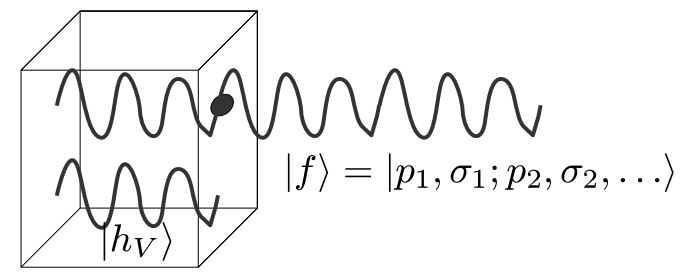

Figure 2: The localized multi-hadronic states $\left|h_{V}\right\rangle$ pertaining to the quantum field problem in a limited region. Asymptotic states $|f\rangle$ are the usual free states characterized by particle momenta and spin components.

The difference between a localized and an asymptotic state is depicted in Fig. 2 . For a single particle in a Non-Relativistic Quantum Mechanics (NRQM) framework, the conceptual difference is easier to grasp: a localized state is described by a wavefunction which vanishes outside the cluster's region whereas an asymptotic state is a wavefunction which is defined over the whole space (e.g. a plane or a spherical wave). In Quantum Field Theory (QFT), a localized state is a state of the Hilbert space defined by the localized problem, e.g. the problem of the quantum field in the cluster's finite region. For a free field, if we enforce fixed or periodic boundary conditions, such states are simply defined by integer occupation numbers for each allowed mode in the finite region, as is well known. For a multiparticle state of non-interacting hadrons, this state will be defined by all occupation numbers of the modes determined by the fields associated with the different species of hadrons, and we will simply denote it with $\left|h_{V}\right\rangle$ (where $h$ stands for "hadrons" and $V$ stands for the finite region of volume $V$ ). On the other hand, an asymptotic state is a state of the Hilbert space defined by the quantum field operators over the whole space; for a free field, these are the familiar multi-particle free states defined by, e.g., momentum and polarization:

$$
|f\rangle=\left|p_{1}, \sigma_{1}, \ldots, p_{N}, \sigma_{N}\right\rangle
$$

There is a noteworthy and deep difference between non-relativistic quantum mechanical and quantum field theoretical case. In the latter, particle number is not fixed and a localized state with multiplicity (defined as the sum of all occupation numbers) $N$ 
does not necessarily correspond to an asymptotic state with particle multiplicity $N 1$. Unlike in NRQM, a localized state with multiplicity $N$ has non-vanishing projections over asymptotic states with different particle multiplicities. In symbols:

$$
|N\rangle_{V}=\alpha_{0, N}|0\rangle+\alpha_{1, N}|1\rangle+\ldots+\alpha_{N, N}|N\rangle+\ldots
$$

with the obvious condition that $\alpha_{i, N} \rightarrow 0$ when $V \rightarrow \infty$ for $i \neq N$. In particular, the vacuum of the finite-region problem $|0\rangle_{V}$ is different from the vacuum of the full-space problem $|0\rangle$, which is commonly known as the Casimir effect. With a straightforward mapping of the Hilbert space of the localized quantum field onto the full Hilbert space, it is possible to express destruction operators of the localized field as linear combinations of destruction and creation operators of the field defined over the whole space [8]. These relations would be sufficient to calculate the coefficients of the above equation, but this is not really needed in the SHM, as it will be soon clear.

\subsection{The Formalism: Basics}

Let us consider a cluster and assume first that it can be described as a mixture of states. Then, the basic postulate implies that the corresponding density matrix is a sum over all localized states projected onto the initial cluster's quantum numbers:

$$
\hat{\rho} \propto \sum_{h_{V}} \mathrm{P}_{i}\left|h_{V}\right\rangle\left\langle h_{V}\right| \mathrm{P}_{i} \equiv \mathrm{P}_{i} \mathrm{P}_{V} \mathrm{P}_{i}
$$

where $\left|h_{V}\right\rangle$ are multi-hadronic localized states and $\mathrm{P}_{i}$ is the projector onto the cluster's initial conserved quantities: energy-momentum, intrinsic angular momentum and its third component, parity and the generators of inner symmetries of strong interactions 2 .

The operator $\mathrm{P}_{i}$ can be formally defined as the projector onto an irreducible vector of the full symmetry group and worked out in a group theory framework [9, 10]. It can be factorized into a "kinematic" projector, associated to general space-time symmetry, and a projector for inner symmetries. For the space-time symmetry, the relevant group is the extended orthochronous Poincaré group $\mathrm{IO}(1,3)^{\uparrow}$ and an irreducible state is defined by a four-momentum $P$, a spin $J$ and its third component $\lambda$ and a discrete parity quantum number $\pi= \pm 1$. Therefore:

$$
\mathrm{P}_{i}=\mathrm{P}_{P, J, \lambda, \pi} \mathrm{P}_{\text {inner }}
$$

If the projector $\mathrm{P}_{P, J, \lambda}$ is worked out in the cluster's rest frame where $P=(M, \mathbf{0})$, it further factorizes into the product of simpler projectors [9, 11], i.e.:

$$
\mathrm{P}_{P, J, \lambda, \pi}=\delta^{4}(P-\hat{P}) \mathrm{P}_{J, \lambda} \frac{\mathrm{I}+\pi \hat{\Pi}}{2}
$$

\footnotetext{
${ }^{1}$ We note that the multiplicity of an asymptotic free state is the properly defined number of particles

${ }^{2}$ Operators in the Hilbert space will be denoted with a hat. Exceptions to this rule are projectors, which will be written in serif font, i.e. P.
} 
where $\hat{P}$ is the four-momentum operator, $\mathrm{P}_{J, \lambda}$ is a projector onto $\mathrm{SU}(2)$ irreducible states $|J, \lambda\rangle$ and $\hat{\Pi}$ is the space reflection operator.

As clusters are color singlets by definition, the projector $\mathrm{P}_{\text {inner }}$ involves flavor and baryon number conservation. In principle, the largest symmetry group one should consider is $\mathrm{SU}(3)$, plus three other $\mathrm{U}(1)$ groups for baryon number, charm and beauty conservation. However, SU(3) symmetry is badly broken by the mass difference between strange and up, down quarks, so it is customary to take a reduced $\mathrm{SU}(2) \otimes \mathrm{U}(1)$ where $\mathrm{SU}(2)$ is associated with isospin and $\mathrm{U}(1)$ with strangeness. The isospin $\mathrm{SU}(2)$ symmetry is explicitly broken as well, but the breaking term is small and can generally be neglected. However, most calculations in the past have replaced isospin $\mathrm{SU}(2)$ with another U(1) group for electric charge, so that the symmetry scheme, from an original $\mathrm{SU}(2)_{\text {isospin }} \otimes \mathrm{U}(1)_{\text {strangeness }} \otimes \mathrm{U}(1)_{\text {baryon }}$ reduces to $\mathrm{U}(1)_{\text {charge }} \otimes \mathrm{U}(1)_{\text {strangeness }} \otimes \mathrm{U}(1)_{\text {baryon }}$.

Altogether, $\mathrm{P}_{\text {inner }}$ can be written as

$$
\mathrm{P}_{\text {inner }}=\mathrm{P}_{I, I_{3}} \mathrm{P}_{\mathrm{Q}} \mathrm{P}_{\chi}
$$

where $I$ and $I_{3}$ are isospin and its third component, $\mathbf{Q}=\left(Q_{1}, \ldots, Q_{M}\right)$ is a vector of $M$ integer abelian charges (baryon number, strangeness, etc.) and $\mathrm{P}_{\chi}$ is the projector onto C-parity, which makes sense only if the system is completely neutral, i.e. $I=0$ and $\mathbf{Q}=\mathbf{0}$; in this case, $\mathrm{P}_{\chi}$ commutes with all other projectors.

From the density matrix (3) the probability of observing an asymptotic multiparticle state $|f\rangle$ is

$$
p_{f} \propto\left\langle f\left|\mathrm{P}_{i} \mathrm{P}_{V} \mathrm{P}_{i}\right| f\right\rangle
$$

which is well-defined in terms of positivity and conservation laws. In fact, (7) is manifestly positive definite and $p_{f}=0$ if the state $|f\rangle$ has not the same quantum numbers as the initial state. By summing over all states $|f\rangle$, one obtains the trace of the operator $\mathrm{P}_{i} \mathrm{P}_{V} \mathrm{P}_{i}$ which is

$$
\sum_{f} p_{f} \propto \operatorname{tr}\left(\mathrm{P}_{i} \mathrm{P}_{V} \mathrm{P}_{i}\right)=\operatorname{tr}\left(\mathrm{P}_{i}^{2} \mathrm{P}_{V}\right)=a \operatorname{tr}\left(\mathrm{P}_{i} \mathrm{P}_{V}\right)
$$

The constant $a$ is divergent and positive. It can be directly checked by choosing the $|f\rangle$ as momentum eigenstates and using the expression on the right hand side of (5). The reason for its presence is the non-compactness of the Poincaré group, which makes it impossible to have a properly normalized projector. The last trace in (8) can be written as

$$
\operatorname{tr}\left(\mathrm{P}_{i} \mathrm{P}_{V}\right)=\sum_{h_{V}}\left\langle h_{V}\left|\mathrm{P}_{i}\right| h_{V}\right\rangle \equiv \Omega
$$

which is, by definition the microcanonical partition function [8], i.e. the sum over all localized states projected onto the conserved quantities defined by the selected initial state. If only energy and momentum conservation is enforced, $\Omega$ takes on a more familiar form:

$$
\Omega=\sum_{h_{V}}\left\langle h_{V}\left|\delta^{4}(P-\hat{P})\right| h_{V}\right\rangle .
$$


Although the mixture of states defined by Eq. (3) allows us to calculate probabilities of any measurement unambiguously, a cluster could in principle also be described by a pure quantum state. Actually, the mixture of states only expresses our ignorance about the true state of the system, which is in principle a pure one, or, more precisely, a pure state entangled with pure states of other clusters. To avoid slipping into fundamental quantum mechanics problems of decoherence and measurement, we take a pragmatic stance here. It suffices to realize that in some low-energy collision events, only one cluster might be created whose state is then necessarily a pure one. According to the postulate of the SHM, this must be an even superposition of all localized states with the initial conserved quantities, i.e.

$$
|\psi\rangle=\sum_{h_{V}} c_{h_{V}} P_{i}\left|h_{V}\right\rangle \quad \text { with }\left|c_{h_{V}}\right|^{2}=\text { const . }
$$

The probability of observing a final state $|f\rangle$ is then

$$
\begin{aligned}
& |\langle f \mid \psi\rangle|^{2}=\left|\sum_{h_{V}}\left\langle f\left|\mathrm{P}_{i}\right| h_{V}\right\rangle c_{h_{V}}\right|^{2} \\
= & \operatorname{const} \sum_{h_{V}}\left|\left\langle f\left|\mathrm{P}_{i}\right| h_{V}\right\rangle\right|^{2}+\sum_{h_{V} \neq h_{V}^{\prime}}\left\langle f\left|\mathrm{P}_{i}\right| h_{V}\right\rangle\left\langle h_{V}^{\prime}\left|\mathrm{P}_{i}\right| f\right\rangle c_{h_{V}} c_{h_{V}^{\prime}}^{*} .
\end{aligned}
$$

If the coefficients $c_{h_{V}}$ have random phases, the last term in Eq. (12) vanishes and we are left with the same expression appearing in Eq. (7); in other words an effective mixture description is recovered. Hence a new hypothesis is introduced in the SHM here: if the cluster is a pure state, the superposition of multi-hadronic localized states must have random phases.

Now the main goal of the model is to determine the probabilities (7) which involves the calculation of the projector $\mathrm{P}_{V}=\sum_{h_{V}}\left|h_{V}\right\rangle\left\langle h_{V}\right|$, a more limited task than the explicit calculation of all scalar products $\left\langle h_{V} \mid f\right\rangle$. Since the states $\left|h_{V}\right\rangle$ are a complete set of states of the Hilbert space $H_{V}$ for the localized problem, the above projector is simply a resolution of the identity of the localized problem and can be written in the basis of the field states. For a real scalar field this is

$$
\mathrm{P}_{V}=\int_{V} \mathcal{D} \psi|\psi\rangle\langle\psi|
$$

where $|\psi\rangle \equiv \otimes_{\mathbf{x}}|\psi(\mathbf{x})\rangle$ and $\mathcal{D} \psi$ is the functional measure; the index $V$ means that the functional integration must be performed over the field degrees of freedom in the region $V$, that is $\mathcal{D} \psi=\prod_{\mathbf{x} \in V} d \psi(\mathbf{x})$. One has to face several conceptual subtleties in the endeavor of calculating the probabilities (77) with the projector (13), e.g. how to deal with field boundary conditions and with their values outside the region $V$. However, by enforcing the known non-relativistic limit is possible to come to an unambiguous and consistent result [8]. 


\subsection{Rates of Multiparticle Channels}

According to the formulae introduced in the previous section, the decay rate of a cluster into a channel $\left\{N_{j}\right\}\left(\left\{N_{j}\right\}\right.$ is the array of multiplicities $\left(N_{1}, \ldots, N_{K}\right)$ for hadron species $1, \ldots, K)$ is proportional to the right hand side of (7) integrated over momenta and summed over polarization states of the final hadrons. Taking into account only energymomentum conservation, so that the projector (5) reduces to

$$
\mathrm{P}_{P J \lambda \pi} \rightarrow \mathrm{P}_{P}=\delta^{4}(P-\hat{P}),
$$

and neglecting quantum statistics effects, this is proportional to the microcanonical partition function with fixed particle multiplicities $[9,8]$

$$
\Omega_{\left\{N_{j}\right\}}=\frac{V^{N}}{(2 \pi)^{3 N}}\left(\prod_{j=1}^{K} \frac{\left(2 S_{j}+1\right)^{N_{j}}}{N_{j} !}\right) \int d^{3} p_{1} \ldots \int d^{3} p_{N} \delta^{4}\left(P_{0}-\sum_{i} p_{i}\right)\left\langle 0\left|\mathrm{P}_{V}\right| 0\right\rangle .
$$

Here $N$ is the number of particles, $S_{j}$ the spin, $P_{0}=(M, \mathbf{0}), M$ is the mass and $V$ is the cluster's proper volume. This formula is the same as it would be obtained in NRQM, with the factor $\left\langle 0\left|\mathrm{P}_{V}\right| 0\right\rangle$ (which becomes 1 in the limit $V \rightarrow \infty$ ) being the only effect of the field theoretical treatment [8]. Since only relative rates make sense, this common factor for all channels is irrelevant.

Loosely speaking, Eq. (14) tells us that the decay rate of a massive cluster into some multi-hadronic channel is proportional to its phase space volume. However, it should be emphasized that the "phase space volume" in (14) is calculated with the measure $d^{3} x d^{3} p$ for each particle, and not with the one usually understood in QFT, i.e. $d^{3} p / 2 \varepsilon$. Although this is also commonly known as "phase space", it is quantitatively different from the properly called phase space measure $d^{3} x d^{3} p$ and should be called "invariant momentum space" measure [12].

Eq. (14) can be cast in a form which makes its Lorentz invariance apparent. Define a four-volume $\Upsilon=V u$ [12] where $V$ is the cluster's rest frame and $u$ its four-velocity vector. Then (14) can be rewritten as:

$$
\begin{aligned}
\Omega_{\left\{N_{j}\right\}}= & \frac{1}{(2 \pi)^{3 N}}\left(\prod_{j=1}^{K} \frac{\left(2 S_{j}+1\right)^{N_{j}}}{N_{j} !}\right) \int d^{4} p_{1} \ldots \int d^{4} p_{N} \\
& {\left[\prod_{i=1}^{N} \Upsilon \cdot p_{i} \delta\left(p_{i}^{2}-m_{i}^{2}\right) \theta\left(p_{i}^{0}\right)\right] \delta^{4}\left(P_{0}-\sum_{i} p_{i}\right)\left\langle 0\left|\mathrm{P}_{V}\right| 0\right\rangle }
\end{aligned}
$$

which is manifestly covariant. In this form it can be directly compared with the general formula for the decay rate of a massive particle into a $N$-body channel:

$$
\Gamma_{N} \propto \sum_{\sigma_{1}, \ldots, \sigma_{N}} \frac{1}{(2 \pi)^{3 N}}\left(\prod_{j} \frac{1}{N_{j} !}\right) \int \frac{d^{3} p_{1}}{2 \varepsilon_{1}} \ldots \int \frac{d^{3} p_{N}}{2 \varepsilon_{N}}\left|M_{f i}\right|^{2} \delta^{4}\left(P_{0}-\sum_{i} p_{i}\right)
$$


where $\sigma$ labels, as usual, polarization states. Comparing (14) with (16) we can infer a dynamical matrix element for the SHM which is

$$
\left|M_{f i}\right|^{2} \propto \prod_{i=1}^{N} \Upsilon \cdot p_{i}
$$

Therefore, according to the SHM the dynamics in cluster decay is limited to a common factor for each emitted particle, which linearly depends on the cluster's spacial size. The four-volume $\Upsilon$ is simply proportional to the four-momentum of the cluster through the inverse of energy density $\rho$ and therefore:

$$
\left|M_{f i}\right|^{2} \propto \frac{1}{\rho^{N}} \prod_{i=1}^{N} P \cdot p_{i} .
$$

This expression explicitly shows the separation between the kinematic arguments of the dynamical matrix element, and the scale $1 / \rho$ which determines particle production. This ought to be ultimately related to the fundamental scale of quantum chromodynamics, $\Lambda_{\mathrm{QCD}}$.

It has already been stressed that the finite cluster size is the distinctive feature of the SHM. This peculiarity of the model stands out when taking into account quantum statistics for the calculation of decay rates. Our final result, for which (14) is a special case when all particles belong to different species, reads

$$
\begin{aligned}
\Omega_{\left\{N_{j}\right\}} & =\int d^{3} \mathrm{p}_{1} \ldots d^{3} \mathrm{p}_{N} \delta^{4}\left(P_{0}-\sum_{i=1}^{N} p_{i}\right) \prod_{j} \sum_{\left\{h_{n_{j}}\right\}} \frac{(\mp 1)^{N_{j}+H_{j}}\left(2 S_{j}+1\right)^{H_{j}}}{\prod_{n_{j}=1}^{N_{j}} n_{j}^{h_{n_{j}}} h_{n_{j}} !} \\
& \times \prod_{l_{j}=1}^{H_{j}} F_{n_{l_{j}}}\left\langle 0\left|\mathrm{P}_{V}\right| 0\right\rangle
\end{aligned}
$$

where $\left\{h_{n_{j}}\right\}$ is a partition of the integer $N_{j}$ in the multiplicity representation, that is $\sum_{n_{j}=1}^{N_{j}} n_{j} h_{n_{j}}=N_{j}, \sum_{n_{j}=1}^{N_{j}} h_{n_{j}}=H_{j}$ and $\sum_{j} N_{j}=N$. The factor $F_{n_{l_{j}}}$ in Eq. (19) are Fourier integrals:

$$
F_{n_{l}}=\prod_{i_{l}=1}^{n_{l}} \frac{1}{(2 \pi)^{3}} \int_{V} d^{3} \mathrm{xe}^{i \mathbf{x} \cdot\left(\mathbf{p}_{c_{l}\left(i_{l}\right)}-\mathbf{p}_{i_{l}}\right)}
$$

over the cluster's region $V, c_{l}$ being a cyclic permutation of order $n_{l}$. The expression (19) has been obtained in refs. [9, 8] and is a generalization of a similar one calculated by Chaichian, Hagedorn and Hayashi [12] whose validity is restricted to large volumes. It is a so-called cluster decomposition of the microcanonical partition function of the channel. For sufficiently large volumes, all terms in Eq. (19) turn out to be proportional to the $H_{j}$ th power of the volume $V[9]$, so that the leading term is the one with $H_{j}=N_{j}$ for all $j$, which leads precisely to Eq. (14). Thus, Eq. (19) is a generalization of (14) containing all corrections due to quantum statistics. 
In general, with respect to the Boltzmann case (14), the channel rate is enhanced by the presence of identical bosons and suppressed by that of fermions. This means that Bose-Einstein and Fermi-Dirac correlations are built into the SHM. The reader has probably anticipated this fact through the appearance of typical Fourier integrals in the cluster decomposition. This feature of the SHM is an almost obvious consequence of the cluster's finite spacial size.

\subsection{Interactions}

So far, we have dealt with non-interacting particles. However, the localized hadronic fields we have used to calculate transition probabilities do interact and this must be taken into account. The energy of the interacting system must be conserved until the final asymptotic multi-hadronic state is reached which is made of particles stable under strong interactions, namely pions, kaons, nucleons and octet hyperons.

Formally, this implies that the projector (5) must include the interacting Hamiltonian in the $\delta^{4}(P-\hat{P})$ operator ${ }^{3}$. The definition (17) for the probability to observe an asymptotic state $|f\rangle$, has to be modified by the insertion of Møller operator $\hat{\Omega}$, yet summing over the complete set of states yields the same result as in Eq. (8) :

$$
\sum_{f} p_{f} \propto \operatorname{tr}\left(\mathrm{P}_{i} \mathrm{P}_{V} \mathrm{P}_{i}\right)=a \operatorname{tr}\left(\mathrm{P}_{i} \mathrm{P}_{V}\right)=a \sum_{h_{V}}\left\langle h_{V}\left|\mathrm{P}_{i}\right| h_{V}\right\rangle \equiv a \Omega
$$

where $a$ is an irrelevant divergent constant and $\Omega$ is the microcanonical partition function of the interacting hadronic system.

An outstanding theorem by Dashen, Ma and Bernstein (DMB) [14] allows us to calculate the microcanonical partition function of an interacting system in the thermodynamic limit $V \rightarrow \infty$ as the sum of the free one plus a term depending only on the physical scattering matrix. It can be expressed as

$$
\operatorname{tr} \delta^{4}(P-\hat{P})=\operatorname{tr} \delta^{4}\left(P-\hat{P}_{0}\right)+\frac{1}{4 \pi i} \operatorname{tr}\left[\delta^{4}\left(P-\hat{P}_{0}\right) \hat{\mathcal{S}}^{-1} \frac{\stackrel{\leftrightarrow}{\partial}}{\partial E} \hat{\mathcal{S}}\right]
$$

where $\hat{P}$ includes the full interaction Hamiltonian, whereas $\hat{P}_{0}$ only contains the free one; $\mathcal{S}$ is the reduced scattering matrix on the energy-momentum shell. If more conserved quantities other than energy and momentum are involved, like those encountered in section 2.2 , the theorem is readily extended and relevant projectors can be placed next to the $\delta$-functions in Eq. (22); it suffices that these conserved quantities are associated with symmetries of both free and interacting theory.

This theorem is indeed the starting point of the hadron-resonance gas model since it can be shown that if only the resonant part of the scattering matrix is retained and the background interaction can be neglected, the main contribution of the second term

\footnotetext{
${ }^{3}$ In all virtually known field theories, there is no additional interacting term for momentum and angular momentum [13].
} 

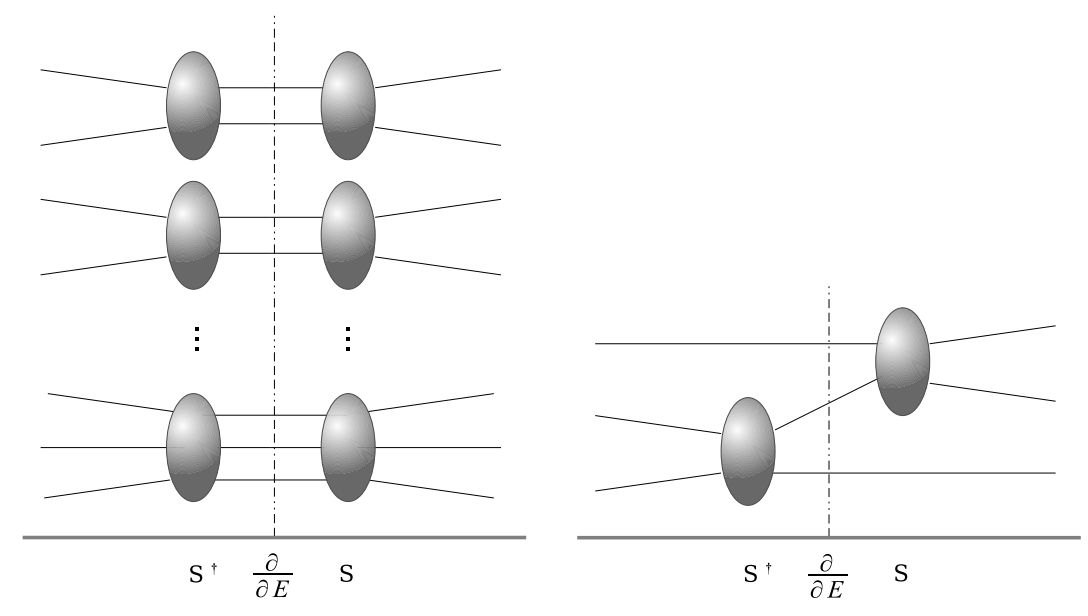

Figure 3: Left panel:symmetric diagrams for the cluster decomposition of the interaction term in the DMB theorem. Right panel: non-symmetric diagrams.

on the right hand side of Eq. (22) is equivalent to considering all hadronic resonances as free particles with distributed mass. More specifically, if a cluster decomposition of the two scattering operators is carried out in Eq. (22), the corresponding diagrams can be divided in two sets: the symmetric diagrams (see Fig. 3, left panel) and the nonsymmetric ones (see Fig. 3, right panel). Taking into account that the terminal legs on both sides have to be the same stable particles on entry and exit (we are calculating a trace), it can be shown that the main contribution to symmetric diagrams comes from the matching resonances in bubbles facing each other. For each term of the trace, this amounts to adding the decay products of resonances considered as free particles with masses distributed according to a relativistic Breit-Wigner form. In symmetric diagrams, there is in principle an additional contribution from resonance interference, which might be non-negligible in case of wide, overlapping resonances with the same decay channel, but it depends on mostly unknown complex parameters and it is thus neglected.

Likewise, the asymmetric diagrams give an additional contribution which also depends on the aforementioned complex interference parameters. While the number of such diagrams greatly exceeds the symmetric ones due to the large number of resonances, contributing terms can be both positive and negative and hopefully a partial cancellation occurs when summing them up for a selected final state.

Altogether, retaining only the resonant interaction and symmetric diagrams in the scattering matrix cluster decomposition, and neglecting resonance interference leads to the following picture: an interacting hadron gas is, to a good approximation, a gas of non-interacting free hadrons and resonances. Since non-resonant interaction should be negligible, the ideal hadron-resonance gas picture holds if the energy density or temperature of the system is large enough for most resonances to be excited. A quantitative assessment of how large these parameters are is still missing, a rough estimate being 
$T>100 \mathrm{MeV}$.

An important remark is now in order. The DMB theorem affirms the equality of two traces, but not of single trace terms. Yet, the decomposition of Eq. (22), implying the ideal hadron-resonance gas picture, is widely used for the calculation of inclusive stable hadronic multiplicities as well, which requires a condition stronger than the equality of the traces on both sides. In other words, using the decomposition (22) to calculate average multiplicities or fluctuations requires the equality to hold for multiparticle generating functions and not only for microcanonical partition functions. Up to now, the extension of (22) to generating functions has never been proved; most likely, it does not hold and corrections to this assumption are necessary. Moreover, while the theorem requires the thermodynamic limit, it is commonly used at finite volume. These limitations should be always kept in mind when using the ideal hadron-resonance gas model.

\subsection{High Energy Collisions}

As we have seen in Sect. 2.3, each individual cluster produced in a high energy collision (shown in Fig. 19), should be hadronized according to formula (19), or its approximation (14), which yields the rates of a given channel within the microcanonical ensemble, including energy-momentum conservation. If clusters are large enough, the microcanonical ensemble could be well approximated by the canonical [9, 15] or even grand-canonical ensemble for average multiplicities. This is not the case in elementary collisions $\left(\mathrm{e}^{+} \mathrm{e}^{-}\right.$, pp, etc.) while it is generally possible in heavy ion collisions, as we will see later in this section. Calculating observables in high energy collisions within the SHM then implies summing microcanonical averages over all produced clusters and this requires in turn knowledge of their charges and four-momenta. In fact, this latter information is unknown to the SHM and only a dynamical model of the pre-hadronization stage of the process (such as, e.g., HERWIG) can provide it.

However, if we are interested in calculating Lorentz-invariant observables (such as average multiplicities) the momenta of clusters are immaterial and only charges and masses matter. In this case, one can introduce a peculiar extra-assumption which allows to considerably simplify the calculation. Basically, it is assumed that the probability distribution:

$$
w\left(\mathbf{Q}_{1}, M_{1} ; \ldots, \mathbf{Q}_{N}, M_{N}\right)
$$

of masses $M$ and conserved abelian charges $\mathbf{Q}$ for $N$ different clusters is the same as one would have by randomly splitting a large cluster (defined as Equivalent Global Cluster, EGC) into $N$ subsystems with given volumes. Thereby, the Lorentz invariant observables can be calculated for one (equivalent global) cluster, whose volume is the sum of proper cluster volumes and whose charge is the sum of cluster charges, hence the conserved charge of the initial colliding system. The full mathematical procedure is described in detail in ref. [16].

In such a global averaging process, the EGC generally turns out to be large enough in mass and volume so that the canonical ensemble becomes a good approximation of the more fundamental microcanonical ensemble [15]; in other words, a temperature 
can be introduced which replaces the a priori more fundamental description in terms of energy density. It was shown that the mass of the EGC should be at least $8 \mathrm{GeV}$ (with an energy density of $0.5 \mathrm{GeV} / \mathrm{fm}^{3}$ ) for the canonical ensemble to be a reasonably good approximation [15. Also, it should be emphasized that in such a mathematical reduction process, temperature has essentially a global meaning and not local as in hydrodynamical models (see next subsection). The only meaningful local quantity in actual physical process are energy densities and individual physical clusters cannot be described in terms of a temperature, unless they are sufficiently large. Nevertheless, this "global" temperature closely mirrors the value of energy density at which clusters hadronize. Indeed, it is this latter value which mainly determines hadronization-related observables; the requirement of the charge distribution of EGC is a side-assumption which is important to simplify calculations, but it can possibly be replaced by other distributions leaving final results essentially unchanged.

In this approach, the primary multiplicity of each hadron species $j$ is given by [16]:

$$
\left\langle n_{j}\right\rangle^{\text {primary }}=\frac{V T\left(2 S_{j}+1\right)}{2 \pi^{2}} \sum_{n=1}^{\infty} \gamma_{S}^{N_{s} n}(\mp 1)^{n+1} \frac{m_{j}^{2}}{n} \mathrm{~K}_{2}\left(\frac{n m_{j}}{T}\right) \frac{Z\left(\mathbf{Q}-n \mathbf{q}_{j}\right)}{Z(\mathbf{Q})}
$$

where $V$ is the (mean) volume and $T$ the temperature of the equivalent global cluster. Here $Z(\mathbf{Q})$ is the canonical partition function depending on the initial abelian charges $\mathbf{Q}=(Q, N, S, C, B)$, i.e., electric charge, baryon number, strangeness, charm and beauty, respectively; $m_{j}$ and $S_{j}$ are the mass and the spin of the hadron $j, \mathbf{q}_{j}=$ $\left(Q_{j}, N_{j}, S_{j}, C_{j}, B_{j}\right)$ its corresponding charges; the upper sign applies to bosons and the lower sign to fermions.

The parameter $\gamma_{S}$ in (23) is an extra phenomenological factor implementing an ad hoc suppression of hadrons with $N_{s}$ strange valence quarks with respect to the equilibrium value. This parameter is outside a pure thermodynamical framework and it is needed to reproduce the data, as we will see. For temperature values of $160 \mathrm{MeV}$ or higher, Boltzmann statistics, corresponding to the term $n=1$ only in the series (23), is a very good approximation (within 1.5\%) for all hadrons but pions. For resonances, the formula (23) is folded with a relativistic Breit-Wigner distribution for the mass $m_{j}$. The final multiplicities, to be compared with the data, are determined by adding to the primary multiplicity (23) the contribution from the decay of unstable heavier hadrons, according to the formula

$$
\left\langle n_{j}\right\rangle=\left\langle n_{i}\right\rangle^{\text {primary }}+\sum_{k} \operatorname{Br}(k \rightarrow j)\left\langle n_{k}\right\rangle
$$

The canonical partition function can be expressed as a multi-dimensional integral

$$
\begin{aligned}
& Z(\mathbf{Q})=\frac{1}{(2 \pi)^{N}} \int_{-\pi}^{+\pi} d^{N} \phi \mathrm{e}^{i \mathbf{Q} \cdot \boldsymbol{\phi}} \\
& \times \exp \left[\frac{V}{(2 \pi)^{3}} \sum_{j}\left(2 S_{j}+1\right) \int d^{3} p \log \left(1 \pm \gamma_{s}^{N_{s j}} \mathrm{e}^{-\sqrt{p^{2}+m_{j}^{2}} / T_{i}-i \mathbf{q}_{j} \cdot \phi}\right)^{ \pm 1}\right]
\end{aligned}
$$




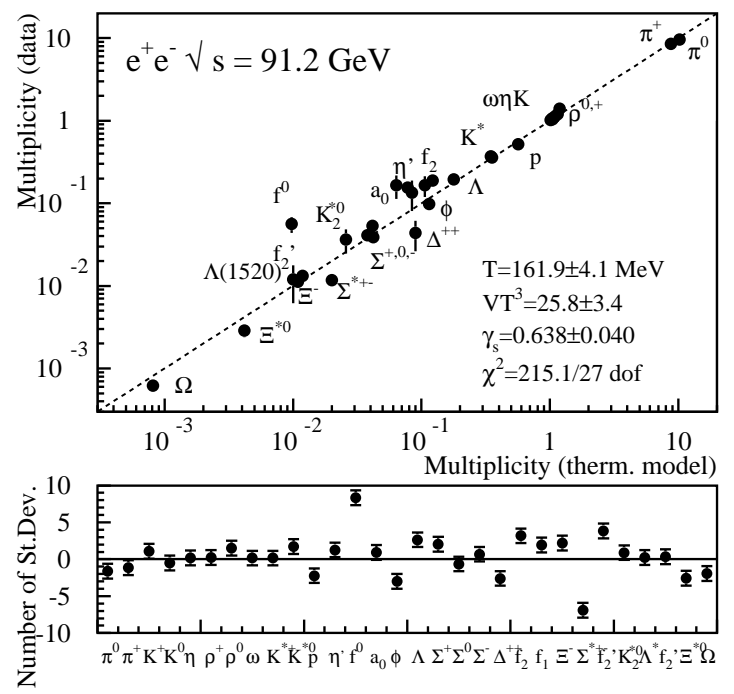

Figure 4: Upper panel: measured vs theoretical multiplicities of light-flavoured hadrons in $\mathrm{e}^{+} \mathrm{e}^{-}$collisions at $\sqrt{s}=91.25 \mathrm{GeV}$. Lower panel: fit residuals (from ref. [17]).

where $N$ is the number of conserved abelian charges. Unlike the grand-canonical case, the logarithm of the canonical partition function does not scale linearly with the volume. Therefore, the so-called chemical factors $Z\left(\mathbf{Q}-n \mathbf{q}_{j}\right) / Z(\mathbf{Q})$ [18] turn out to be less than unity even for a completely neutral system at finite volume (canonical suppression) and reach their grand-canonical value 1 at asymptotically large volumes [19, 4].

The light-flavoured multiplicities in $\mathrm{e}^{+} \mathrm{e}^{-}$show a very good agreement with the predictions of the model, as it shown in Fig. 4: the temperature value is about $160 \mathrm{MeV}$ and the strangeness undersaturation parameter $\gamma_{S} \sim 0.7$. Similar good agreements are found for many kinds of high energy elementary collisions over a large energy range [4. Also, an excellent agreement between measured and predicted relative abundances of heavy flavoured hadronic species in $\mathrm{e}^{+} \mathrm{e}^{-}$collisions by using the model parameters fitted to light-flavoured multiplicities [4, 17, as shown in Table 1.

The overall striking feature is that the temperature turns out to be approximately constant over two orders of magnitude in centre-of-mass energy with a value of 160$170 \mathrm{MeV}$ (see Fig. 5) and very close to the QCD critical temperature as determined from lattice calculations. There must certainly be a profound connection between the thus-found hadronization temperature and QCD thermodynamics, a connection which has not been made clear yet. Nevertheless, this finding indicates that hadronization is a universal process occurring at a critical value of the local energy density, i.e. when clusters have an energy density of $\simeq 0.5 \mathrm{GeV} / \mathrm{fm}^{3}$.

The parameter $\gamma_{S}$ is found to be less than 1 in all examined elementary collisions, ranging from $\sim 0.5$ in hadronic collisions to $\sim 0.7$ in $\mathrm{e}^{+} \mathrm{e}^{-}$collisions (see Fig. 9). This 


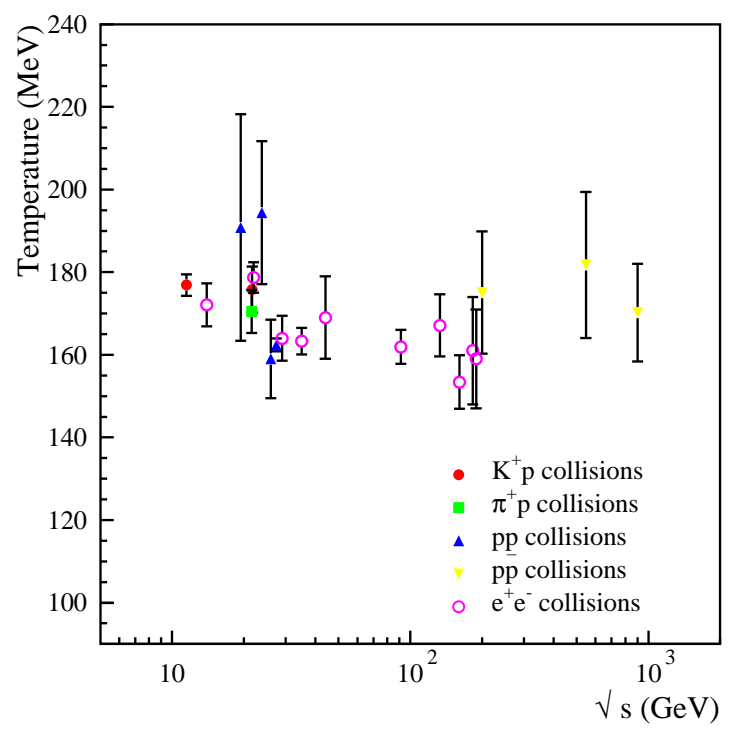

Figure 5: Temperatures fitted in elementary collisions as a function of center-of-mass energy.

extra parameter most likely reflects the different mass of the strange quark with respect to lighter $\mathrm{u}, \mathrm{d}$ quarks. This is a second scale, besides $\Lambda_{Q C D}$, which must play a role in hadronization in view of its value $\mathcal{O}(100) \mathrm{MeV}$. Altogether, one can say that the SHM description of hadronization is in excellent agreement with QCD at least with regard to the the number of parameters. The two parameters $T$ and $\gamma_{S}$ correspond to the two fundamental scales $\Lambda_{Q C D}$ and $m_{s}$, the strange quark mass. While we lack a definite relation connecting them (see, however ref. [17]), it is worth stressing that a phenomenological description of hadronization in terms of fewer parameters cannot be possible.

Finally, the statistical model shows a very good capability of reproducing transverse momentum spectra in hadronic [16] as well as heavy ion collisions [20]. Particularly, the phenomenon of approximate $m_{T}$ scaling observed in pp collisions is nicely accounted for by the model. However, the exact $\mathbf{p}_{T}$ conservation at low energy and the increasing importance of jet emission at high energy restrict the validity of the statistical canonical formulae to a limited centre-of-mass energy range. Within this region, a clear consistency is found between the temperature parameter extracted from the spectra and that from average multiplicities. Altogether, this finding bears out one of the key predictions of the SHM, namely the existence of a definite relation between the dependence of particle production rates on mass and, for each particle species, their momentum spectra (in the cluster's rest frame) because they are both governed by one parameter, the energy density (or temperature) at hadronization. 


\begin{tabular}{|c|c|c|c|c|}
\hline Particle & Experiment $(\mathrm{E})$ & Model $(\mathrm{M})$ & Residual & $(M-E) / E[\%]$ \\
\hline$D^{0}$ & $0.559 \pm 0.022$ & 0.5406 & -0.83 & -3.2 \\
$D^{+}$ & $0.238 \pm 0.024$ & 0.2235 & -0.60 & -6.1 \\
$D^{*+}$ & $0.2377 \pm 0.0098$ & 0.2279 & -1.00 & -4.1 \\
$D^{* 0}$ & $0.218 \pm 0.071$ & 0.2311 & 0.18 & 6.0 \\
$D_{1}^{0}$ & $0.0173 \pm 0.0039$ & 0.01830 & 0.26 & 5.8 \\
$D_{2}^{* 0}$ & $0.0484 \pm 0.0080$ & 0.02489 & -2.94 & -48.6 \\
$D_{s}$ & $0.116 \pm 0.036$ & 0.1162 & 0.006 & 0.19 \\
$D_{s}^{*}$ & $0.069 \pm 0.026$ & 0.0674 & -0.06 & -2.4 \\
$D_{s 1}$ & $0.0106 \pm 0.0025$ & 0.00575 & -1.94 & -45.7 \\
$D_{s 2}^{*}$ & $0.0140 \pm 0.0062$ & 0.00778 & -1.00 & -44.5 \\
$\Lambda_{c}$ & $0.079 \pm 0.022$ & 0.0966 & 0.80 & 22.2 \\
\hline$B_{s}$ & $0.399 \pm 0.011$ & 0.3971 & -0.18 & -0.49 \\
$B^{*} / B(\mathrm{uds})$ & $0.098 \pm 0.012$ & 0.1084 & 0.87 & 10.6 \\
$\left.B^{* *} \times B^{0} B\left({ }^{*}\right) \pi\right)$ & $0.749 \pm 0.040$ & 0.6943 & -1.37 & -7.3 \\
$\left(B_{2}^{*}+B_{1} \times B R\left(B\left(^{*}\right) \pi\right)\right.$ & $0.090 \pm 0.018$ & 0.0800 & -0.57 & -26.7 \\
$B_{s 2}^{*} \times B^{*} B(B)$ & $0.0093 \pm 0.0024$ & 0.00631 & -1.24 & -11.4 \\
$\mathrm{~b}-\mathrm{baryon}$ & $0.103 \pm 0.018$ & 0.09751 & -0.30 & -5.3 \\
$\Xi_{b}^{-}$ & $0.011 \pm 0.006$ & 0.00944 & -0.26 & -14.2 \\
\hline \hline
\end{tabular}

Table 1: Abundances of charmed hadrons in $\mathrm{e}^{+} \mathrm{e}^{-} \rightarrow \mathrm{c} \overline{\mathrm{c}}$ annihilations and bottomed hadrons in $\mathrm{e}^{+} \mathrm{e}^{-} \rightarrow \mathrm{b} \overline{\mathrm{b}}$ annihilations at $\sqrt{s}=91.25 \mathrm{GeV}$, compared to the prediction of the statistical model (from ref. [17]).

\subsection{Heavy Ion Collisions}

In heavy ion collisions, the system is much larger and two possibilities are usually envisaged: either hadronizing clusters are simply much larger than those in elementary collisions; or clusters are hydrodynamical cells, i.e. they are small but in thermal contact with each other due to previous thermalization, which implies a strong correlation between their position and momentum and charge densities (see Fig. 6). In both case the canonical or grand-canonical formalisms apply to individual clusters. For the former case, it is worth mentioning that the transition from a canonical to a grand-canonical description effectively occurs when the cluster volume is of the order of $100 \mathrm{fm}^{3}$ at an energy density of $0.5 \mathrm{GeV} / \mathrm{fm}^{3}$ [18]. If the EGC reduction assumption still applies, chemical factors in Eq. (23) are replaced by fugacities, and in this case the phase-space integrated multiplicities read

$$
\left\langle n_{j}\right\rangle^{\text {primary }}=\frac{V T\left(2 S_{j}+1\right)}{2 \pi^{2}} \sum_{n=1}^{\infty} \gamma_{S}^{N_{s} n}(\mp 1)^{n+1} \frac{m_{j}^{2}}{n} \mathrm{~K}_{2}\left(\frac{n m_{j}}{T}\right) \exp \left[n \boldsymbol{\mu} \cdot \mathbf{q}_{j} / T\right] .
$$




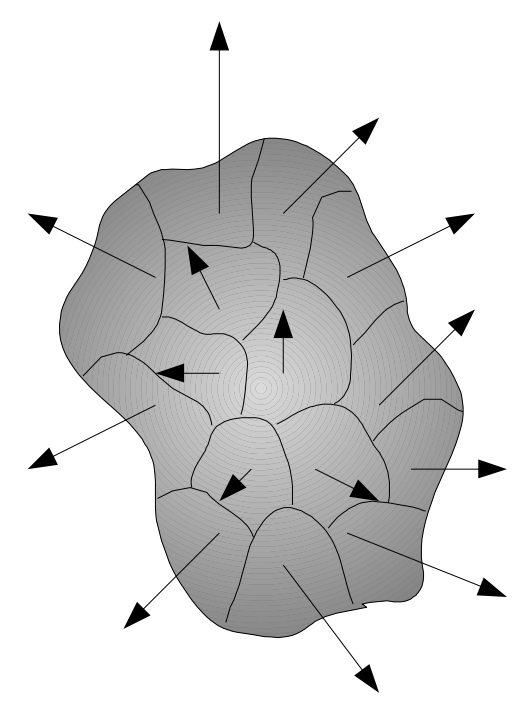

Figure 6: Spacial distributions of clusters in heavy ion collisions according to the hydrodynamical picture. In this model, nearby clusters interact from an early stage on and their momenta and charges are strongly correlated with their positions, unlike in elementary collisions.

$\boldsymbol{\mu}$ is a vector of chemical potentials pertaining to the conserved abelian charges, i.e. the electrical chemical potential $\mu_{Q}$, the baryon chemical potential $\mu_{B}$ and the strangeness chemical potential $\mu_{S}$. Usually, but not always, $\mu_{S}$ and $\mu_{Q}$ are determined by enforcing strangeness neutrality and by fixing the ratio $Q / B$ to be the same as the initial $Z / A$ ratio of the colliding nuclei.

In the framework of the hydrodynamical model, formula (26) applies to individual clusters identified with hydrodynamic cells and both temperature and chemical potentials depend on space-time; when integrating particle densities to get average multiplicities, one should take into account this dependence. It is important to stress that the hydrodynamical description is a salient feature of heavy ion collisions due to the early thermalization of the system in the partonic phase, a phenomenon which does not occur in elementary collisions. It is this early thermalization which establishes the strong correlation between positions and velocities of clusters, supposedly absent in elementary collisions.

Provided that rapidity distributions are wide enough, and that there is little variation of the thermodynamical parameters of clusters around midrapidity, the formula (26) describes rapidity densities of hadrons at midrapidity as well: this condition is fulfilled at RHIC energies, but not at AGS and SPS energies, where the measured rapidity distributions are not significantly wider than those of a single fireball at the temperature found $[22]$.

In general, the fits to particle multiplicities in heavy ion collisions are of the same good quality as in elementary collisions (see Fig. 7). Many groups have analyzed the 


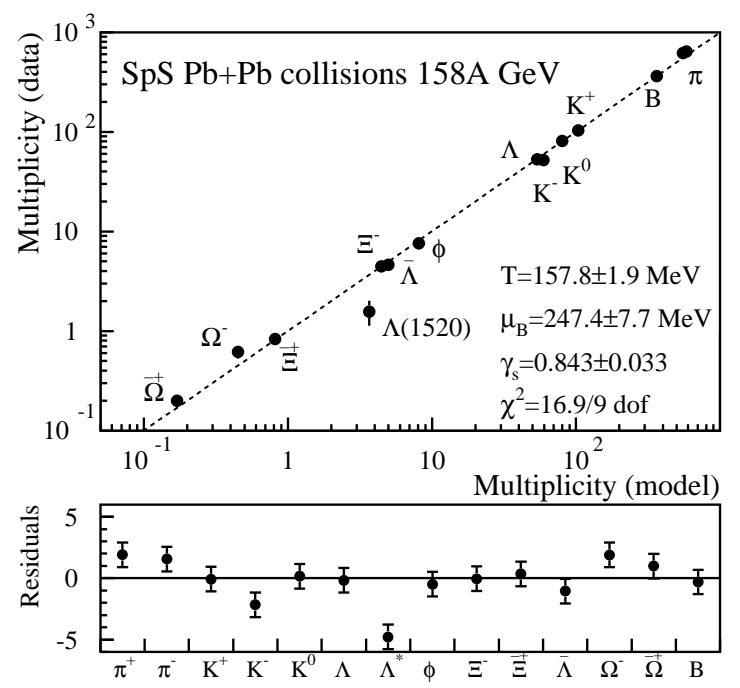

Figure 7: Upper panel: measured vs theoretical multiplicities of light-flavoured hadrons in $\mathrm{Pb}-\mathrm{Pb}$ collisions at $\sqrt{s}_{N N}=17.2 \mathrm{GeV}$. Lower panel: fit residuals (from ref. [21]).

data over more than a decade [3] and the overall description is very good throughout all explored energies and one finds a smooth curve in the $T-\mu_{B}$ plane (see Fig. 8).

\subsection{Strangeness Production}

The statistical model is a very useful tool to study one of the main features of relativistic heavy-ion collisions, the increase of relative strangeness production with respect to elementary collisions. This was one of the early signatures proposed for Quark-Gluon Plasma formation [24], and it has therefor attracted much attention both on the theoretical and experimental side. According to the SHM, this is mainly an effect of the increase of the global volume from elementary to heavy-ion collisions. In elementary collisions, the EGC volume is small enough for the chemical factors (see Eq. (25)) of strange particles to be consistently less than 1 for systems with vanishing net strangeness, a phenomenon known as strangeness canonical suppression.

However, canonical suppression is not enough to account for strangeness enhancement from pp to heavy-ion collisions: also an increase of $\gamma_{S}$ is needed. This is demonstrated by neutral mesons containing strange quarks, especially $\phi$ meson, which do not suffer canonical suppression but are relatively more abundant in heavy-ion collisions [25, 26]. Therefore, from a SHM viewpoint, one can say that, as far as particle abundances is concerned, the only substantial difference between elementary and heavy ion collisions resides in the different $\gamma_{S}$ values, which are generally higher in heavy-ion collisions and increase slowly as a function of center-of-mass energy (see Fig. 9): at RHIC 


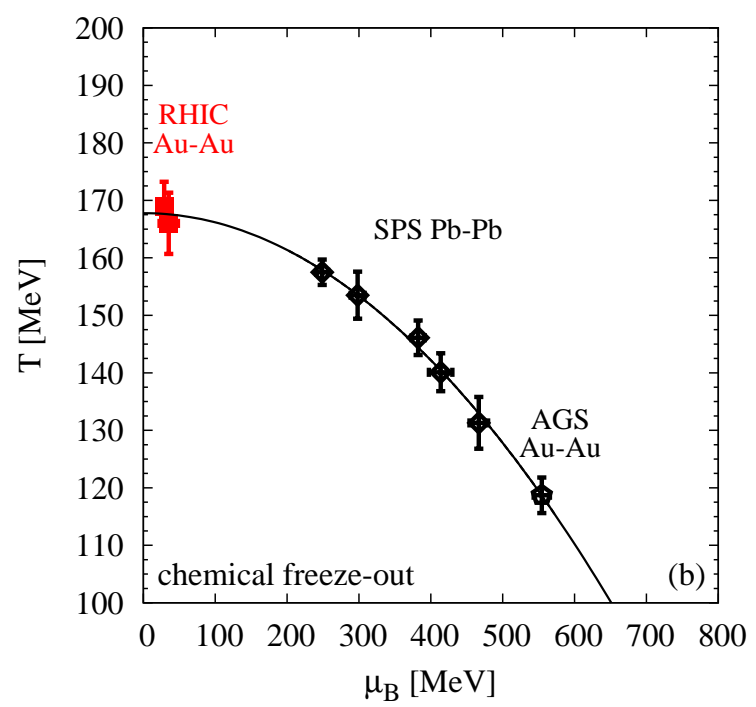

Figure 8: Temperature vs baryon chemical potential fitted with multiplicities in heavy ion collisions (from ref. [23]).

energies one finds $\gamma_{S} \simeq 1$ in central collisions. However, since $\gamma_{S}$ is an empirical parameter which lies outside of a pure statistical mechanics framework, this observation does not clarify the origin of strangeness enhancement.

It is interesting to note that, while $\gamma_{S}$ shows no special regularity in elementary collisions, the ratio of newly produced $\bar{s} s$ pairs over one half $\bar{u} u+\bar{d} d$ pairs (the socalled Wroblewski ratio $\lambda_{S}$ ) turns out to be around $0.2-0.25$ at all energies, whereas it is definitely higher in heavy ion collisions (see Fig. 10).

These differences, both in $\gamma_{S}$ and $\lambda_{S}$ have been and still are subject of investigation. Relevant information comes from the centrality dependence of strangeness production, which provides an interpolation from single pp collisions at large values of nuclear impact parameter to head-on heavy-ion collisions at low values thereof. The enhancement has been measured by the experiments WA97 and NA57 at SPS energy [27] and STAR at RHIC [28] for hyperons and other strange particles and it has been found to be hierarchical in strangeness content (highest for $\Omega^{-}$, lowest for $\Lambda$ ). These observations led some authors [29] to put forward a picture where $\gamma_{S}$ is an effective parametrization of a canonical suppression. For large enough baryon number and charge, it is possible to take a mixed canonical-grand-canonical approach where only strangeness conservation is enforced, while electric and baryon-chemical potential are introduced. The chemical factors $Z\left(S-S_{j}\right) / Z(S)$ depend on the volume and saturate at large volumes, as expected. Therefore, if we want to account for $\gamma_{S}<1$ with this mechanism, there must be some small sub-regions within a large fireball where strangeness is exactly vanishing even for the most central collisions. Thereby, chemical factors are significantly less than 1 and a suppression with respect to the grand-canonical limit is implied. However, this model 

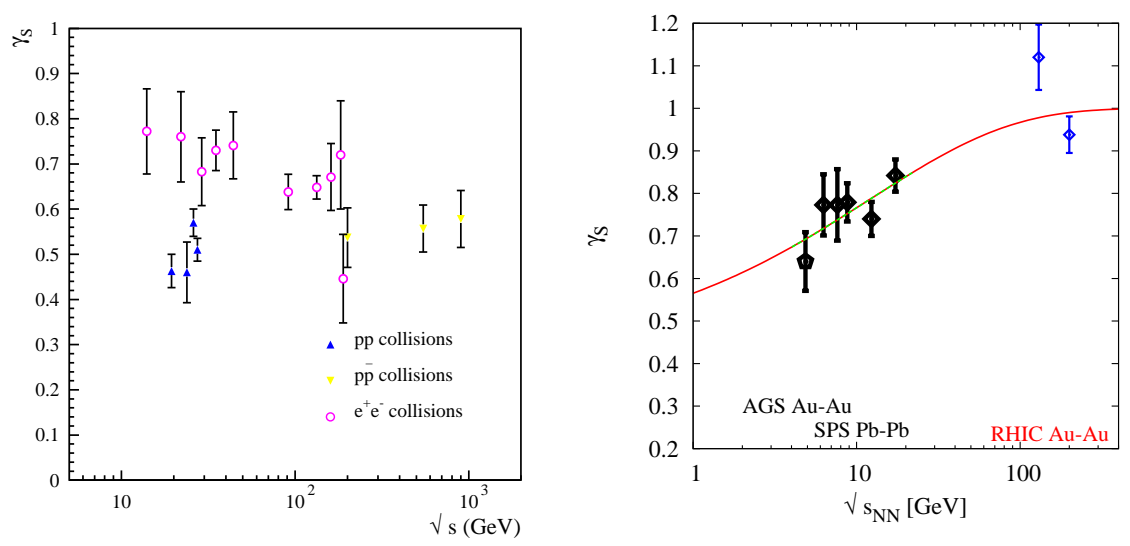

Figure 9: Left panel: the strangeness undersaturation parameter $\gamma_{S}$ as a function of center-of-mass energy in $\mathrm{e}^{+} \mathrm{e}^{-}$, pp and $\mathrm{p} \overline{\mathrm{p}}$ collisions. Right panel: the strangeness undersaturation parameter $\gamma_{S}$ as a function of center-of-mass energy in central heavy ion collisions (from ref. [23]).

has two major problems:

1. Since measured enhancement steadily increases from peripheral to central collisions and hadronization temperature does not change [30, 31, 23], the volume of the sub-regions with $S=0$ should also increase and a saturation is thus expected (see Fig. 11); yet, no saturation is observed, which is quite an oddity.

2. As has been mentioned, canonical suppression has no effect on $\phi$; yet, the relative yield of this meson with two constituent strange quarks is also observed to increase from peripheral to central collisions [32] and with $\gamma_{S}=1$ and the observed constant temperature, this cannot occur.

Recently, a geometrical explanation of these two features has been advocated [33] based on a superposition of emission from a hadron-resonance gas at full chemical equilibrium with $\gamma_{S}=1$, defined as the core, and from nucleon-nucleon collisions at the boundary of the overlapping region of the two colliding nuclei, defined as the corona, from which produced particles escape unscathed. Since in NN collisions strangeness is suppressed with respect to a fully equilibrated, grand-canonical hadron gas, if such NN collisions account for a significant fraction of total particle production, a global fit to particle multiplicities will find $\gamma_{S}<1$, as indeed observed in data. The idea of superposing different sources is common to other models (a similar one is discussed in ref. [34]). The peculiar feature of this specific model is to assume single NN collisions as secondary sources; only in this case does it seem possible to reproduce centrality dependence of the $\phi$ meson. 


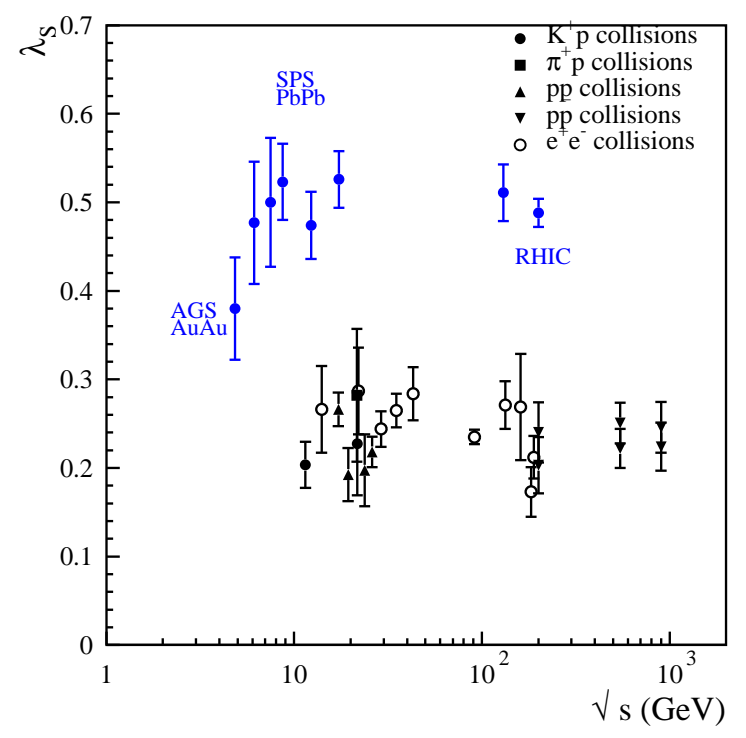

Figure 10: Wroblewski ratio $\lambda_{S}$ (see text for definition) as determined in elementary and heavy ion collisions from fits of multiplicities to the statistical hadronization model.

\subsection{Thermalization: How Is It Achieved?}

After discussing the success of the SHM in reproducing particle multiplicities and the intriguing universality of its main parameter, the temperature, one is obviously led to the question how this can come about. A classical process of thermalization through binary collisions between formed hadrons, advocated in heavy ion collisions [35], is ruled out in elementary collisions because the expansion rate is fast and hadrons are not interacting for a long enough time for this to take place. But even in heavy ion collisions peculiar features of the data cannot be explained in a hadronic kinetic picture [36] without invoking the predominance of multi-body collisions; since, in this case, the hadronic mean free path is comparable or smaller than Compton wavelengths, the collisional picture breaks down naturally.

There is evidence that thermalization occurs at a relatively early stage over a large region (i.e. clusters several femtometers wide) in heavy ion collisions, whereas it is a late phenomenon (i.e. very close to hadronization) occurring over small (of the order of $1 \mathrm{fm}$ ) distances in elementary collisions. Yet, the agreement between model and data is surprisingly accurate in elementary collisions, even more accurate than in heavy ion collisions, the only difference being in the level of strangeness phase space saturation. Somehow, the hadrons must be born into equilibrium as Hagedorn first pointed out [37] and was reaffirmed by others [38, 39].

The idea that this thermal-like behavior is of genuine quantum-mechanical origin and not related to semi-classical collision processes, is shared by many [39] and is es- 


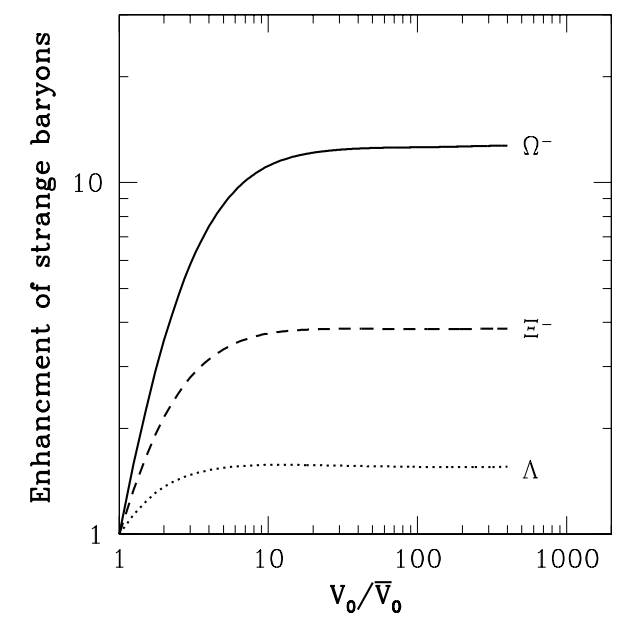

Figure 11: Canonical enhancement (defined here as the ratio between the chemical factor $Z\left(S-S_{j}\right) / Z(S)$ and its value at some fixed volume $\left.V_{0}\right)$ as a function of volume for hyperons (from ref. [29]).

poused in this review. A different point of view was presented in ref. [40] where it was argued that the thermal behavior could just be mimicked by a matrix element which is weakly dependent on the final kinematic variables in (16), a scenario called "phase space dominance". But even this scenario requires stringent conditions on the dependence of cluster decay rates on the channel multiplicity (essentially like $A^{N}$ ) [10] such that the exponential dependence of production rates on mass is not spoiled. Hence phase-space dominance is not less trivial in any way. A possible path to distinguish between the two scenarios is provided by the analysis of exclusive rates at low energy, although it must be pointed out that the observed identical particle correlations already favors SHM which is endowed with a built-in spacial extension, unlike phase-space dominance.

However, whether it is a proper thermal-statistical equilibrium in a finite volume or rather a phase space dominance effect, there must be a profound reason behind this phenomenon, which ultimately has to be related to the nature of QCD as a theory with strong coupling at large distances. Also, we believe that the intriguing universality of the temperature found in elementary collisions as well as heavy ion collisions and its resemblance of the QCD critical temperature is not accidental.

If we assume that post-hadronization collisions are unable to restore equilibrium, how can a quantum evolution process ensure it? Several years ago it was pointed out that a closed quantum system whose classical counterpart is chaotic and ergodic can give rise to thermal distributions provided that the so-called Berry conjecture applies [41. Berry's conjecture [42] essentially states that the high-lying eigenfunction amplitudes $\psi(\mathbf{x})$ in configuration space appear to be random Gaussian numbers and, as a consequence, momentum space distribution is microcanonical [43]. This "quantum thermalization" mechanism has been invoked to explain the observed thermal-like distributions in hadronic processes [44]. Of course, this argument requires that classical 
QCD is chaotic (as it has indeed been advocated [45]), that Berry's conjecture holds for quantum fields, and that it can be applied to a dynamical process like hadronization. All of these conditions are non-trivial, but pursuing these ideas further may give rise to interesting developments.

Recently, another appealing idea to explain the universality of thermal features in multihadron production has been put forward [46]. It invokes an analogy between confinement and black hole physics. It is conjectured that the phenomenon of confinement is equivalent to the formation of an event horizon for colored signals (quarks and gluons). Similarly to Hawking-Unruh radiation, the spectrum of hadrons, emitted as the result of a high energy collision, is thermal because no information can be conveyed from the causally disconnected region beyond event horizon. According to this so-called Hawking-Unruh scheme of hadronization, temperature is related to the string tension and is thereby universal. Another interesting consequence of this idea is that the extra strangeness suppression observed in elementary collisions can be quantitatively explained [17] as an effect of the different strange quark mass.

These attempts relating the observed thermal features in hadron production processes to quantum chaos or Hawking-Unruh radiation are still in a developmental stage. Whether they will keep their promises will be seen in the future. Certainly, both share the vision that there is a fundamental quantum mechanical mechanism behind this phenomenon and no (or little) room for a classical collisional thermalization process.

\section{Quark Recombination}

The statistical hadronization model provides a successful description of key features of hadron production without explicitly invoking the underlying fundamental degrees of freedom in QCD, quarks and gluons. Clusters inherit the characteristics of partons from which they emerge, but it seems that there is no explicit role for hadronic substructure in determining final hadron ratios except for the extra strangeness suppression parameter $\gamma_{S}$ which is a direct manifestation of the differences between $\mathrm{u}, \mathrm{d}$ and $\mathrm{s}$ quark masses. On the other hand, many hadronization models involve parton degrees of freedom explicitly and it seems that some observables in relativistic heavy ion collisions at very high energy require the assumption of an underlying parton dynamics.

In a parton-based approach the goal is to calculate the probability to produce a set of hadrons $h_{1}, \ldots, h_{n}$ from a given partonic "initial" state $\mathcal{C}$. In general, this problem involves interactions of partons at a scale around $\Lambda_{\mathrm{QCD}}$ which, as emphasized before, is a highly non-perturbative problem and as yet unsolved. In defining this problem, we have to overcome another obstacle which is connected to the preparation of the partonic "initial" state $\mathcal{C}$. Quarks and gluons are not asymptotic states. Rather, they usually appear as intermediate states in the scattering reaction $A+B \rightarrow \mathcal{C}+X \rightarrow h_{1}+\ldots h_{n}+X^{\prime}$. Hence the partonic state $\mathcal{C}$ is difficult to "prepare", and in fact the nature of quantum field theory requires us to integrate over all possible states $\mathcal{C}$. In the worst case, $\mathcal{C}$ might not be well defined at all because the partonic state couples to the other states, $A, B$, 
$X$, etc. in a non-factorizable way. In fact, this will always be true for any state $\mathcal{C}$ which is not a color singlet, since the final hadron ensemble $h_{1}, \ldots, h_{n}$ is colorless and color must be exchanged with other parts of the system during hadronization. We call this problem the factorization problem.

Several factorization schemes for hadronization have been developed starting from the 1970s, resulting in some successful effective descriptions of hadronization. They are usually based on a proof that in a well defined process a simple set of possible intermediate parton states $\mathcal{C}_{i}$ is giving the leading contribution to hadron production in powers of a small expansion parameter. The best known example is single hadron production in $\mathrm{e}^{+} \mathrm{e}^{-}$or hadronic collisions at a large momentum scale $Q$. The leading contribution to the cross section in terms of powers of $1 / Q$ comes from intermediate states with just one single parton $\mathcal{C}_{1}=\{g\}, \mathcal{C}_{2}=\{u\}, \ldots$ and the cross section $\sigma^{h}$ can be factorized into a cross section for producing the intermediate parton $\mathcal{C}_{i}$ and a probability $D_{i / h}$ to produce $h$ from $C_{i}$ [47, 48, 49]

$$
\sigma^{h} \sim \sum_{i} \sigma^{\mathcal{C}_{i}} \otimes D_{i / h}
$$

Note that the leading contribution does not involve an interference effect between different partons in the amplitude and the complex conjugated amplitude and the process $C_{i} \rightarrow h$ can be formulated as a probabilistic problem with a probability distribution $D_{i / h}(z)$ called a fragmentation function. $z$ is the fraction of the original parton momentum carried by the hadron $h$. In general, the functions $D_{i / h}(z)$ can not be calculated from first principles (which would be equivalent to fully solving the hadronization problem). However, it makes single hadron production at large momentum treatable by separating off the hadronization probability for a given hadron which can be measured by experiment and is universally applicable to all processes where such a factorization holds.

All hadronization models involving partons have to start from an assumption about a well defined parton "initial state". The exact details will depend on the process under investigation. Note that the SHM had such a basic axiom as well, defining the probability of multihadron states in a cluster. In this section, we discuss the model of quark recombination or coalescence. It also involves a plausible but ad hoc assumption at the outset. This is less rigorous than the factorization argument that can be given for parton fragmentation, but it is phenomenologically very successful. We will focus on applications of quark recombination to heavy ion collisions.

\subsection{Parton Fragmentation and Its Limitations}

For a better understanding of recombination it is instructive to investigate situations where parton fragmentation is failing.

Factorization of fragmentation functions off a hard parton scattering cross section was first introduced for simple single scale processes like single-inclusive hadron productions [50]. Later the LEP Collider at CERN brought reliable data on hadron production 
in $\mathrm{e}^{+} \mathrm{e}^{-}$collisions which allowed the extraction of universal sets of fragmentation functions 51, 52, 53 (see 49 for a modern review). In a physical gauge fragmentation functions have a straightforward definition in terms of counting operators $a_{h}^{\dagger}(P) a_{h}(P)$ of asymptotic hadron momentum eigenstates applied to the parton field. E.g., if parton $i$ is a quark described by a field $\psi$ then we have

$$
D_{i / h}(z) \sim \int d x e^{-i P x / z}\left\langle 0\left|\psi(0) a_{h}^{\dagger}(P) a_{h}(P) \bar{\psi}(x)\right| 0\right\rangle
$$

where $x$ is a light cone coordinate conjugate to the light cone momentum $P$.

There are many situations where factorization involving a vacuum fragmentation function could not be proven or is explicitly violated. In some situations it is easy to see qualitatively why this is the case. One example is hadron production at very forward rapidity in hadronic collisions. A phenomenon known as the leading particle effect [54] can be observed if relative abundances of hadrons at forward rapidity are considered. The FNAL E791 collaboration [55] found a large asymmetry between $D^{-}$and $D^{+}$mesons in fixed target experiments with $\pi^{-}$beams on nuclei. Obviously a fragmentation from $c$ or $\bar{c}$ quarks or from gluons should be nearly flavor blind and the charm quark mass sets a (semi-)hard scale that can be used to justify perturbative arguments. However, in this case the asymmetry comes from recombination of the $\bar{c}$ from a $c \bar{c}$ pair produced in the collision with a $d$ valence quark from the beam $\pi^{-}$remnants. This mechanism is enhanced compared to the $c+\bar{d}$ recombination which involves only a sea quark from the $\pi^{-}$[56]. Obviously the presence of the beam fragments acting as spectators destroy the favorable conditions for vacuum fragmentation.

The leading particle effect also motivated the birth of the coalescence picture. Das and Hwa developed a model for recombination of partons streaming forward in hadronic collisions [57]. There is no thermalized parton phase in this case, but this simple model has many of the features of the recombination models for RHIC we discuss below:

(1) The input is a (multi-)parton distribution $f\left(p_{1}, \ldots, p_{n}\right)$ whose spectrum and chemical composition remain unchanged during the hadronization process.

(2) Partons coalesce into hadrons according to recombination functions $\Phi$ which play the role of squared hadronic wave functions.

(3) Only valence quarks of the hadrons play a role in the hadronization process.

The number of recombining hadrons is therefore given by

$$
N_{h} \sim \Phi \otimes f\left(p_{1}, \ldots, p_{n}\right)
$$

where $n=2$ or 3 for mesons and baryons respectively. The first condition is usually reinterpreted to mean that the coalescence process is fast enough such that the momentum distribution and chemical composition of the partons do not react to the depletion of partons during recombination. This assumption addresses the question of a welldefined "initial" parton state indicated in the last section. The third condition has been 
the center of many debates. Where are the gluons and sea quarks? While there is no calculation of this process from first principles, we do have some qualitative arguments. First, the momentum transfer involved is rather small and it is questionable whether perturbative partons can be resolved. Second, we know that even in processes that start at large momentum scales, degrees of freedom get frozen and give constituent masses to quarks [58]. We could therefore argue that a dressing of quarks happens before the final recombination step. Third, we have no reason to believe that the hadrons originally formed are indeed free hadron states. There will necessarily be a formation time before we can actually interpret them as asymptotic states. The last two arguments are related to the role of chiral symmetry during hadronization. Chiral symmetry breaking has not been explicitly incorporated in recombination models thus far.

\subsection{The Recombination Formalism}

\subsubsection{Basic Theory}

Coalescence or recombination of particles appears in a wide array of systems in atomic, molecular and plasma physics. As a first approximation the details of the dynamical process are usually ignored. Rather, the adiabatic approximation of a projection of the initial multiparticle state onto the final coalesced state is considered. This instantaneous approximation is widely used in the literature for the case of partons coalescing into hadrons. The formalism introduced here is also related to the successful coalescence model for nucleons [59, 60]. After first applications to forward hadron production and the leading particle effect the coalescence concept was soon applied to heavy ion collisions 61, 62, 63, 64.

The number of hadrons $h$ coalescing from a partonic system characterized by a density matrix $\hat{\rho}$ is given by

$$
N_{h}=\int \frac{d^{3} P}{(2 \pi)^{3}}\langle h ; \mathbf{P}|\hat{\rho}| h ; \mathbf{P}\rangle .
$$

Instantaneous here means that the states are defined on a hypersurface which is typically either taken to be at constant time, $t=$ const., or on the light-cone $t= \pm z$. Note that the notion of instantaneous recombination guarantees that condition (1) from section 3.1 is automatically fulfilled.

The instantaneous projection formalism has the conceptual disadvantage that only three components of the four-momentum are conserved in the underlying $2 \rightarrow 1$ or $3 \rightarrow 1$ coalescence process. Qualitatively this can be corrected by assuming that the participants can scatter off the surrounding particles which can build up or dissipate their virtuality. At least in a equilibrium state this should not change the momentum distribution or chemical composition of the particles. However, no explicit formalism has been developed to include this effect quantitatively.

More dynamical approaches beyond the instantaneous projection approximation have been considered as well [65]. They conserve 4-momentum by allowing a finite 
width for hadrons and will be discussed later. We first focus on instantaneous projection models that were discussed for heavy ion collisions first by Greco, Ko and Lévai [GKL] [66, 67]; Fries, Müller, Nonaka and Bass [FMNB] [68, 69, 70]; Hwa and Yang [HY] [71, 72] and Rapp and Shuryak [RS] [74.

¿From Eq. (29) we can derive an expression for the number of mesons with a certain momentum $\mathbf{P}$ from recombination 69]

$$
\frac{d N_{M}}{d^{3} P}=\sum_{a, b} \int \frac{d^{3} R}{(2 \pi)^{3}} \frac{d^{3} q d^{3} r}{(2 \pi)^{3}} W_{a b}\left(\mathbf{R}-\frac{\mathbf{r}}{2}, \frac{\mathbf{P}}{2}-\mathbf{q} ; \mathbf{R}+\frac{\mathbf{r}}{2}, \frac{\mathbf{P}}{2}+\mathbf{q}\right) \Phi_{M}(\mathbf{r}, \mathbf{q}) .
$$

Here $M$ denotes the meson and $a, b$ are its coalescing valence partons. $W_{a b}$ and $\Phi_{M}$ are the Wigner functions of the partons and the meson respectively, $\mathbf{P}$ and $\mathbf{R}$ are the momentum and spatial coordinate of the meson, and $\mathbf{q}$ and $\mathbf{r}$ are related to the relative momentum and position of the quarks. The sum runs over all possible combinations of quantum numbers of valence quarks in the meson, which is usually replaced by a degeneracy factor $C_{M}$. Eq. (31) is very intuitive for a hadronization hypersurface at constant time. It can be made Lorentz-covariant to allow for relativistic kinematics.

The corresponding formula for baryons, containing 3 valence quarks, is completely analogous [69]. Note that Eq. (31) implements principle (3) from Section 3.1] by taking into account only the lowest Fock state of the meson. It has been attempted to generalize Eq. (31) to include more partons which would be gluons or pairs of sea quarks, accounting for the next terms in a Fock expansion [73]. There is no difficulty in including these in the model, but data on elliptic flow scaling in heavy ion collisions puts bounds on the size of contributions from higher Fock states.

Let us recall the definition of the Wigner function for a meson consisting of two quarks

$$
\Phi_{M}(\mathbf{r}, \mathbf{q})=\int d^{3} s e^{-i \mathbf{s} \cdot \mathbf{q}} \varphi_{M}\left(\mathbf{r}+\frac{\mathbf{s}}{2}\right) \varphi_{M}^{*}\left(\mathbf{r}-\frac{\mathbf{s}}{2}\right)
$$

where the 2-quark meson wave function in position space $\varphi_{M}$ can be represented as

$$
\left\langle\mathbf{r}_{1} ; \mathbf{r}_{2} \mid M ; \mathbf{P}\right\rangle=e^{-i \mathbf{P} \cdot\left(\mathbf{r}_{1}+\mathbf{r}_{2}\right) / 2} \varphi_{M}\left(\mathbf{r}_{1}-\mathbf{r}_{2}\right)
$$

The Wigner function of the partons can be defined in a similar way from the density matrix $\hat{\rho}$ [69]. For further evaluation the Wigner functions have to be specified. Usually, the parton Wigner function $W_{a b}$ is replaced by its classical limit, the multi-parton phase space distribution $f\left(x_{1}, p_{1} ; \ldots ; x_{n}, p_{n}\right)$ on the hypersurface of hadronization. The Wigner functions of the hadrons are not well known in this particular case. In general the wave function of a hadron depends in a non-trivial way on the frame of reference, the particular process, and the resolution scale. In heavy ion collisions the resolution scale is non-perturbative, and of the order of the temperature at hadronization, $T_{c}<200$ $\mathrm{MeV}$. Therefore the hadron Wigner functions are often modeled ad hoc. Luckily the dependence of observables in heavy ion collisions on the shape of the wave function is suppressed as we will discuss below. 
The different groups mentioned above using instantaneous recombination mainly differ in details of how they apply the basic equation (31) to nucleus-nucleus and hadronnucleus collisions [75]. We will discuss some of their unique features and refer the reader to the original literature and reviews [75, 76] for further details.

\subsubsection{Different Implementations of Instantaneous Recombination}

In the implementation by Greco, Ko and Lévai [GKL] [66, 67] the full overlap integral in Eq. (31) over both relative position and momentum of the partons is calculated with Monte-Carlo techniques. In their case the Wigner function of the hadron is a simple product of spheres in position and momentum space with radii correlated through the uncertainty relation.

In the implementations by Fries, Müller, Nonaka and Bass [FMNB] [68, 69, 70]; Hwa and Yang [HY] [71, 72 and Rapp and Shuryak [RS] [74 Eq. (31) is simplified by integrating out the information about position space. This leads to a pure momentum space formalism in which the information about the hadron is further compressed into a squared (momentum space) wave function. In a frame of reference in which one momentum component of the hadron is much larger than the others the two momentum components transverse to the hadron momentum can be integrated out as well, leading to a simple 1-dimensional overlap integral. Such a formalism is appropriate, e.g., for hadron production at large transverse momentum in the laboratory frame. It is also equivalent to the original formulation by Das and Hwa using recombination functions [57]. The yield of mesons with momentum $P$ can then be expressed as

$$
\frac{d N_{M}}{d^{3} P}=C_{M} \int_{\Sigma} \frac{d \sigma \cdot \mathbf{P}}{(2 \pi)^{3}} \int_{0}^{1} d x_{1} d x_{2} \Phi_{M}\left(x_{1}, x_{2}\right) W_{a b}\left(x_{1} \mathbf{P} ; x_{2} \mathbf{P}\right)
$$

where $d \sigma$ is the hypersurface of hadronization. In many cases the emission integral over the hypersurface is not calculated explicitly, but replaced by a normalization factor proportional to the volume of the hadronization hypersurface. $x_{1}$ and $x_{2}$ are the fractions of momentum carried by the two valence quarks.

For the lowest Fock state of a meson the squared wave function is mostly parameterized as 69]

$$
\Phi_{M}\left(x_{1}, x_{2}\right)=B x_{1}^{\alpha_{1}} x_{2}^{\alpha_{2}} \delta\left(x_{1}+x_{2}-1\right) .
$$

The powers $\alpha_{i}$ determine the shape while the constant $B$ normalizes the integral over $\Phi_{M}$ to unity. Light cone distribution amplitudes suggest $\alpha_{i}=2$ for a light quark system [77, 78, 79]. Values $\alpha_{c}=5, \alpha_{u, d}=1$ are used for the charm-light quark system in $D$ mesons in Ref. [74]. The extreme case of the two quarks exactly sharing the momentum, $\Phi_{M}\left(x_{1}, x_{2}\right)=\delta\left(x_{1}-1 / 2\right) \delta\left(x_{2}-1 / 2\right)$, is often considered for schematic estimates. Formally this corresponds to $\alpha_{i} \rightarrow \infty$ with the ratio of the $\alpha_{i}$ fixed.

\subsubsection{Dynamical Recombination}

One of the drawbacks of the instantaneous approximation is the violation of energy conservation which has to be restored, on average, by interactions in the medium which are 
not further specified. One early attempt to improve the situation was the introduction of a mass distribution for the quarks [80]. The mass distribution can be thought of as an effective way to incorporate some in-medium effects. This approach allows to enforce both momentum and energy conservation and one finds fairly good agreement with data for transverse momentum spectra in heavy ion collisions.

A promising new direction has recently been taken by Ravagli and Rapp (RR) [65, 81. They advocate a dynamical approach in which the instantaneous projection of quark states onto hadron states is replaced by a transport picture which involves ensembles $f_{a}$ and $f_{M}$ of quarks and mesons evolving with time. To determine properties of the meson distribution starting from given quark distributions one solves the Boltzmann equation

$$
\begin{aligned}
\frac{\partial}{\partial t} f_{M}(t, P)=-\frac{\Gamma}{\gamma_{P}} f_{M}(t, P)+\int \frac{d^{3} p_{1} d^{3} p_{2}}{(2 \pi)^{6}} \int d^{3} x f_{a}\left(x, p_{1}\right) f_{b}\left(x, p_{2}\right) & \\
& \times \sigma(s) v\left(p_{1}, p_{2}\right) \delta^{(3)}\left(\mathbf{P}-\mathbf{p}_{1}-\mathbf{p}_{2}\right)
\end{aligned}
$$

with a gain term describing the scattering of a quark-antiquark pair $a$ - $b$ into a meson state $M$ with finite width $\Gamma$ and a corresponding loss term describing the decay of $M$ into a pair $a-b$. Here $\sigma(s)$ is the resonant cross section in Breit-Wigner form with width

$\Gamma, v$ is the relative velocity of the pair and $\gamma_{P}=\sqrt{\mathbf{P}^{2}-m^{2}} / m$ is the gamma factor of the meson with mass $m$.

This implementation naturally conserves 4-momentum and permits, e.g., the study of kinetic energy scaling [82, 81]. The problem of baryon formation is slightly more difficult in a transport approach and has not yet been addressed. We should also note that even for a finite meson width the creation of light Goldstone bosons, in particular pions, is still problematic. They are much lighter than the sum of the participating quark masses which are usually taken to be constituent quark masses.

\subsection{Connection with other Hadronization Models}

The recombination model was conceived as a correction to vacuum fragmentation in order to include multi-parton effects. Indeed, for a first, intuitive understanding it is helpful to view parton fragmentation and parton recombination as two limiting cases of hadronization. The former fixes the parton state $\mathcal{C}$ to be a single parton in the vacuum. The latter assumes the parton state to be a subset of a populated, maybe even thermalized phase space, such that valence partons are readily available. This simple picture allows a basic understanding of most qualitative features of both mechanisms.

Recombination is the most effective way to form hadrons from a thermalized parton phase space, at least for not too small momentum $P$. This is easy to see in the instantaneous momentum space formalism, but it can be shown to hold numerically in more general cases as well. Let us assume that the partons at moderate to large values of $p$ (large enough that the energy $E$ can be approximated by $E \approx p$ ) are distributed according to a Boltzmann distribution $f_{a}=e^{-p / T}$. Fragmentation then leads to a hadron spectrum which has an effective slope $\langle z\rangle T$ with $\langle z\rangle<1$ being the mean value of the 
momentum fraction $z$ of the hadron from a fragmentation function $D(z)$. Hence the resulting slope is steeper than $1 / T$. On the other hand, hadrons recombining according to Eq. (35) have a distribution

$$
f_{M} \sim \int d x e^{-x P / T} e^{-(1-x) P / T} \Phi(x) \sim e^{-P / T}
$$

for mesons, and similar for baryons. Hence we conclude that the recombination formalism is indeed more effective for large $P$ as long as the spectrum is exponential.

We also notice that for large momenta $(P>>M)$ we recover the results we obtain from the (momentum dependent) statistical model. At least this is true if the same set of resonances is included. In practice, this has not yet been implemented for recombination. Only a few studies have been dealing with resonances in a limited way [83, 67, 84]. Of course, one can argue that the contributions from resonance decay to stable hadron spectra are formally vanishing in the limit $P \rightarrow \infty$. With this caveat the correspondence between recombination and statistical hadronization would be precise.

Unfortunately, the limit $P \rightarrow \infty$ is an academic one, since in any realistic system the parton spectrum will eventually become a power law $f_{a} \sim p^{-\alpha}$ for increasing momentum $p$. Once this kinematic regime is reached fragmentation becomes more effective, producing hadrons with a slightly shifted power law $\sim P^{-\alpha-\delta}$, where the small exponent $\delta$ approximates the changed slope after applying the fragmentation function. On the other hand the power is doubled or tripled for recombining mesons $\left(\sim P^{-2 \alpha}\right)$ or baryons $\left(\sim P^{-3 \alpha}\right)$, respectively. Hence, in any realistic system fragmentation dominates at asymptotically large momentum, in accordance with QCD factorization theorems.

One might ask whether recombination is interesting to consider given its apparently small region of applicability. Curiously, in heavy ion collisions at RHIC we find a kinematic region at intermediate transverse momentum between 1.5 and $6 \mathrm{GeV} / c$ where recombination still dominates over fragmentation, and the momenta are large enough compared to the masses involved such that the simple instantaneous projection formalism, even without including resonances, gives very reasonable results for hadron spectra and elliptic flow. Moreover, unlike statistical hadronization, recombination can make certain predictions connecting the kinematic behavior of hadrons and quarks. Elliptic flow scaling in heavy ion collisions is a famous example. It must be connected to hadronization because it exhibits a dependence on the number of valence quarks. We will offer a survey of recent experimental results supporting quark recombination further below.

Let us summarize this subsection so far. We have found that there is a formal connection between parton recombination and the statistical hadronization model at large momenta. Without doubt a successful implementation of parton recombination at low momenta in heavy ion collisions should reproduce the statistical model in that kinematic region as well. Such a description is not available yet. It would have to include energy and momentum conservation, at least in a statistical sense, as well as the full range of resonances and their decays. This issue of resonances is also related to entropy conservation. Direct coalescence into stable hadrons seems to reduce the 
number of particles by about a factor two, raising questions about the second law of thermodynamics. At intermediate and large momenta, where the current formalism is applicable, only a minute fraction of the total number of particles is residing, so that entropy is not of any grave concern here.

We conclude this subsection by discussing some further connections between recombination and fragmentation. We return to the original question of preparing a welldefined partonic state $\mathcal{C}$. The fragmentation formalism puts a cut between long- and short-distance physics which is well motivated because we can not calculate the former perturbatively, while we can compute the latter. The single parton state is treated like an observed final state of the perturbative calculation. However we know that many processes during fragmentation are still partonic in nature, not hadronic. Hence we are tempted to ask whether we could define a partonic state $\mathcal{C}^{\prime}$ later in the development of the jet parton shower. A jet shower might include a dozen or more partons which at some point feel the pressure from the QCD vacuum to bind into hadrons. This binding might well be described again by recombination.

It is not at all clear that such a parton ensemble in a jet "just before" hadronization can always be well defined. However, the cluster picture of statistical hadronization seems to support this idea. One can try to choose this as a starting assumption. This picture of the fragmentation process has been implemented in the recombination model of Hwa and Yang [85]. Instead of fragmenting hard partons directly, they define the parton shower of a jet (initiated by a hard parton) through so-called shower distributions. These are given by non-perturbative splitting functions $S_{i / j}(z)$ which describe the probability to find a parton of flavor $j$ with momentum fraction $z$ in a jet originating from a hard parton $i$. The parton content of a single jet is then allowed to recombine and should match the corresponding fragmentation functions. E.g. for a meson $M$ with valence quarks $a$ and $b$

$$
D_{i / M}(z)=\int d x_{a} d x_{b}\left[S_{i / a}\left(x_{a}\right) S_{i / b}\left(\frac{x_{b}}{1-x_{a}}\right)+(a \leftrightarrow b)\right] \Phi_{M}\left(x_{a} / z, x_{b} / z\right) .
$$

The shower distributions are not determined from first principles but are fitted to reproduce the known fragmentation functions for pions, protons and kaons [85].

The power of this approach lies in the fact that the fragmentation part of the hadron spectrum is computed with the same recombination formalism that is applied to the thermalized part of phase space. It is then very natural to allow coalescence of shower partons with thermal or soft partons [72] by defining a total parton distribution

$$
f_{a}(p)=f_{a}^{\text {soft }}(p)+f_{a}^{\text {shower }}(p)=: T(p)+S(p) .
$$

which is subject to the recombination formalism. For mesons we obtain two known contributions. The term $\sim S S$ corresponds to the coalescence of two partons from the same jet and reproduces fragmentation, the term $\sim T T$ recombines two soft partons and reproduces the soft recombination spectrum discussed in the previous subsection. The mixed term $\sim T S$ is new and describes the pick-up of a soft parton by a jet parton to 
form a meson. For baryons two of these mixed terms, $\sim T T S$ and $\sim T S S$, exist. These soft-hard coalescence terms make important contributions in some cases. They can, e.g., explain the final state dependence of the Cronin effect observed in $d+A$ collisions at RHIC [86, 87], and lead to jet-like correlations between hadrons [88, 89, 90, 91].

To summarize, there is reason to believe that parton coalescence could be an overarching qualitative concept for hadronization, based on its phenomenological success. However, so far it is only defined in a naive parton model sense. Beyond that it is not clear that the parton content of an arbitrary scattering of hadrons or nuclei before hadronization can be written down in a well-defined way. E.g. in Eq. (39), it is not clear why hadrons forming at very different times in the soft and hard sector can be treated together. It is also not completely understood how the scale dependence of the fragmentation functions can be interpreted correctly [92].

\subsection{Experimental Evidence for Recombination}

We have already discussed the leading particle effect and mentioned the Cronin effect in hadron-nucleus collisions. Here, we want to review some of the support for recombination from heavy ion collisions. Three early results from the Relativistic Heavy Ion Collider led to the conclusion that hadrons at intermediate transverse momentum $p_{T}(1.5 \mathrm{GeV} / c$ $<p_{T}<4 \ldots 6 \mathrm{GeV} / c$ ), are produced by recombination of quarks.

(a) The baryon-to-meson ratios in central Au-Au collisions was found to be enhanced 93 , 94 . The proton/pion ratio $\approx 1$ is incompatible with expectations from fragmentation.

(b) The nuclear modification factors $R_{A A}$ and $R_{C P}$, i.e. the ratio of yields in central $\mathrm{Au}-\mathrm{Au}$ collisions compared to pp and peripheral collisions, resp., scaled by the number of binary nucleon-nucleon collisions, is not suppressed for baryons [93, 95].

(c) The anisotropy of particle production in azimuth relative to the reaction plane, called the elliptic flow parameter $v_{2}$, scales in a universal way for mesons and baryons [95, 96, 97, 98].

\subsubsection{Hadron Spectra and Ratios}

Fig. 12 shows the measured anti-proton/pion 93 and $\bar{\Lambda} / K_{S}^{0}$ 94 ratios as functions of transverse momentum $p_{T}$ for various centralities and collision systems. At intermediate $p_{T}$ the baryon-to-meson ratios in central $\mathrm{Au}-\mathrm{Au}$ collisions are significantly larger than those in $\mathrm{e}^{+} \mathrm{e}^{-}$[99] or pp collisions [100]. These results were the first clear indication that hadronization proceeds differently in $\mathrm{Au}-\mathrm{Au}$ collisions and $\mathrm{pp}$ collisions even in a regime traditionally expected to be dominated by jet fragmentation.

Fig. 13 shows anti-proton/pion (left) and $\bar{\Lambda} / K_{S}^{0}$ (right) ratios compared with instantaneous coalescence calculations from Greco, Ko and Lévai [GKL] [67, 101] and Fries, Müller, Nonaka and Bass [FMNB] [69, 102]. Despite some deviations these calculations 


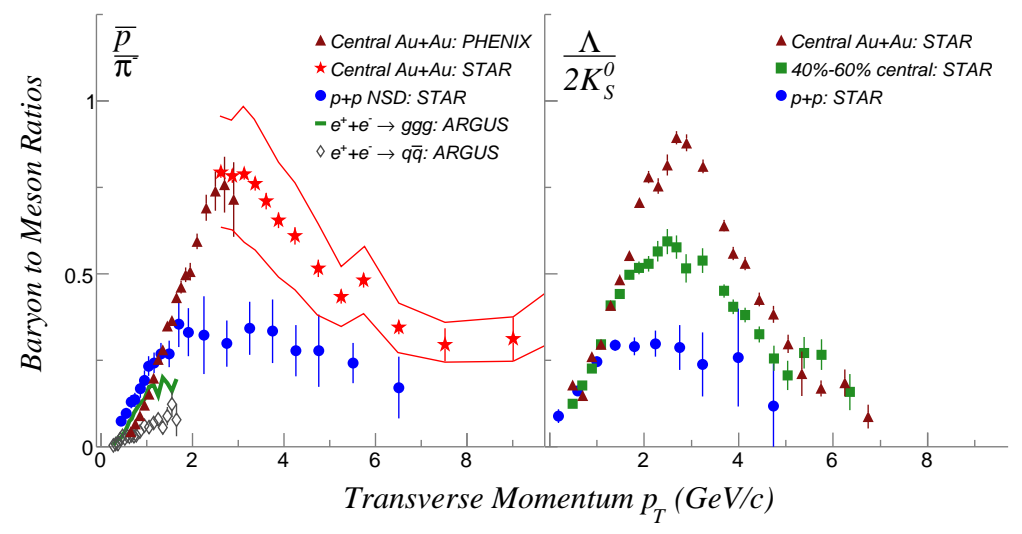

Figure 12: Left: $\bar{p} / \pi^{-}$ratios measured in central Au-Au collisions at $\sqrt{s_{N N}}=200 \mathrm{GeV}$ at RHIC, compared to measurements from $\mathrm{e}^{+} \mathrm{e}^{-}$and pp collisions. Right: The ratio $\bar{\Lambda} / 2 K_{S}^{0}$ for central and mid-central Au-Au collisions at $\sqrt{s_{N N}}=200 \mathrm{GeV}$ measured by STAR. The $\bar{p} / \pi^{-}$ratio from pp collisions from STAR is shown for comparison.

reproduce the characteristic peak structure very well. This peak comes from the interplay of soft production which leads to steeply rising ratios at low $p_{T}$, and low ratios at large $p_{T}$ coming from jet fragmentation. Recombination gives an explanation why soft production dominates out to intermediate $p_{T}$.

The different behavior of baryons and mesons is also expressed in different nuclear suppression factors. Fig. 14 shows the nuclear modification factor $R_{C P}$ measured at RHIC for various identified hadron species. If a nuclear collision is a simple superposition of binary nucleon collisions, $R_{C P}$ will equal one. Therefore the suppression of $R_{C P}$ at large $p_{T}$ is a signature for jet quenching [103]. The baryons $(\Lambda+\bar{\Lambda}, \Xi+\bar{\Xi}$, and $\Omega+\bar{\Omega})$ [94, 104], show significantly less suppression than mesons (kaons or $\phi$ ) [105, 106]). The same behavior was found for protons and pions [107]. The second important observation is that the behavior is universal for all baryons and all meson separately, and largely independent of mass. Heavy $\phi$ mesons behave like pions, not like the equally heavy protons or $\Lambda$ baryons [106, 82. This is a clear indication that the decisive factor here is the number of valence quarks.

Fig. [15 shows spectra from the FMNB coalescence model [69] for neutral pions, kaons, protons and hyperons in central $\mathrm{Au}-\mathrm{Au}$ collisions compared to data from RHIC [104, 107]. The recombination region is visible as an exponential slope at intermediate $p_{T}$ with a transition to a power-law shape at higher $p_{T}$. Note that the FMNB model simply adds recombination and fragmentation contributions. Nevertheless the description of the data is quite good above $2 \mathrm{GeV} / c$. Note that the transition from an exponential shape to a power-law shape happens at a higher $p_{T}$ for baryons than it does for mesons. This is expected and due to the inherent suppression of baryons in vacuum fragmentation which makes coalescence competitive up to quite large $p_{T}$. 


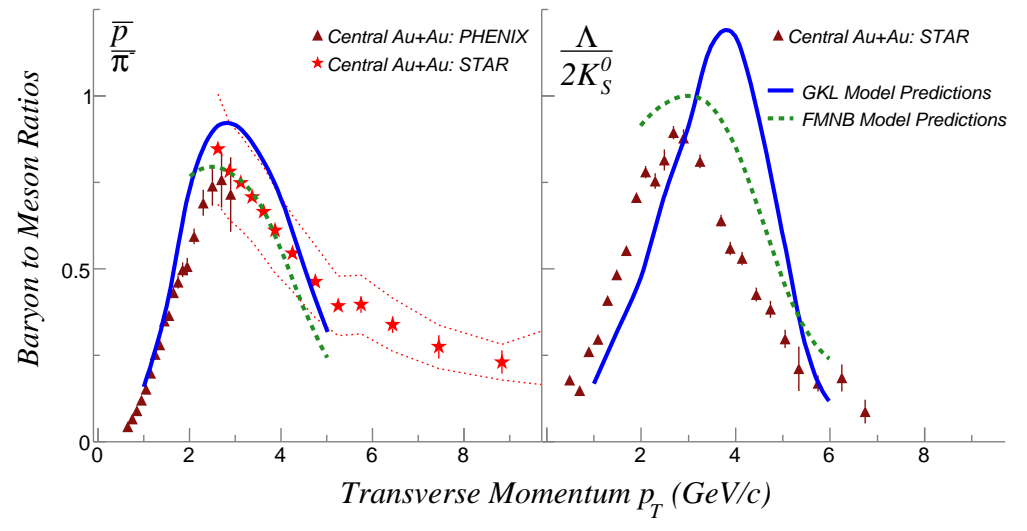

Figure 13: Ratios of baryon yields to meson yields for central $\mathrm{Au}$-Au collisions at 200 GeV. The GKL and FMNB calculations for $\bar{p} / \pi^{-}$(left) and $\Lambda / 2 K_{s}^{0}$ are compared to STAR and PHENIX data.

\subsubsection{Elliptic Flow}

Recombination makes a straightforward prediction for the elliptic flow $v_{2}$ of hadrons [108, using the elliptic flow of quarks as input. This behavior can be easily derived in the instantaneous momentum-space formalism. Let us assume that the elliptic flow of a set of partons $a$ just before hadronization is given by an anisotropy $v_{2}^{a}\left(p_{T}\right)$ at midrapidity. The phase space distribution of partons $a$ can then be written in terms of the azimuthal angle $\phi$ as

$$
f_{a}\left(\mathbf{p}_{T}\right)=\bar{f}_{a}\left(p_{T}\right)\left(1+2 v_{2}^{a}\left(p_{T}\right) \cos 2 \phi\right)
$$

where odd harmonics are vanishing due to the symmetry of the system and higher harmonics are neglected. $\bar{f}$ is the distribution averaged over the azimuthal angle $\phi$.

For a meson with two valence partons $a$ and $b$ and for small elliptic flow $v_{2} \ll 1$ one has 69.

$$
\begin{aligned}
v_{2}^{M}\left(p_{T}\right)= & \frac{\int d \phi \cos (2 \phi) d N_{M} / d^{2} p_{T}}{\int d \phi d N_{M} / d^{2} p_{T}} \\
& \sim \int d x_{a} d x_{b} \Phi_{M}\left(x_{a}, x_{b}\right)\left[v_{2}^{a}\left(x_{a} p_{T}\right)+v_{2}^{b}\left(x_{b} p_{T}\right)\right] .
\end{aligned}
$$

In the case of a very narrow wave function in momentum space $(\alpha \rightarrow \infty)$ this leads to the expression

$$
v_{2}^{M}\left(p_{T}\right)=v_{2}^{a}\left(x_{a} p_{T}\right)+v_{2}^{b}\left(x_{b} p_{T}\right) .
$$

with fixed momentum fractions $x_{a}$ and $x_{b}\left(x_{a}+x_{b}=1\right)$.

Thus for hadrons consisting of light quarks which exhibit the same elliptic flow before hadronization we arrive at a simple scaling law with the number of valence quarks $n$ :

$$
v_{2}^{h}\left(p_{T}\right)=n v_{2}^{a}\left(p_{T} / n\right)
$$




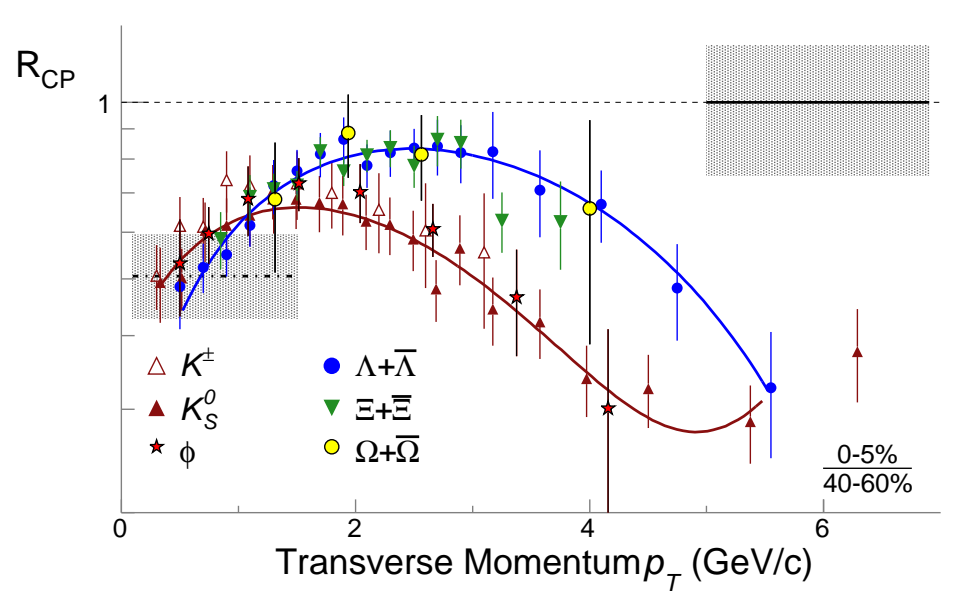

Figure 14: Nuclear modification factors $\left(R_{C P}\right)$ for various identified particles measured in $\mathrm{Au}$-Au collisions at $\sqrt{s_{N N}}=200 \mathrm{GeV}$ by the STAR collaboration. The values for baryons and mesons fall into two separate bands (indicated by lines to guide the eye) with the baryon $R_{C P}$ larger than the meson $R_{C P}$.

This scaling law had first been found in data from RHIC and was quickly interpreted as a coalescence artefact [109. Since then this connection has been solidified in almost all coalescence models [69, 110, 111. Eq. (42) has also been used to extract the elliptic flow of heavy quarks from measurements of $D$ mesons [112, 113]. The line of thought presented for elliptic flow can be extended to harmonics beyond the second order. Generalized scaling laws for the 4th and 6th order harmonics have been derived in Ref. [114].

Fig. 16 shows data on $v_{2}$ scaled by the number $n$ of valence quarks in a given hadron as a function of $p_{T} / n$ for several species of identified hadrons at $\sqrt{s_{N N}}=200$ $\mathrm{GeV}$ [106, 115]. To investigate the quality of agreement, the data from the top panel are scaled by a polynomial fitted to the universal curve and plotted in the bottom panel. At low $p_{T}$ hydrodynamics [116, 117, 118] predicts an ordering of $v_{2}$ by hadron mass which is confirmed by data [95, 97, 119]. That is the reason why valence quark scaling of $p_{T}$ is not working for $p_{T} / n<0.5 \mathrm{GeV} / c$. The two phenomena can be reconciled by first scaling the $v_{2}$ results in transverse kinetic energy $E_{T}=m_{T}-m_{0}$, and then applying a scaling in $n$ [82. The result is also shown in Fig. 16. The scaling at low $m_{T}-m_{0}$ holds to high accuracy.

The experimentally observed scaling is a spectacular success for the recombination picture. However, it is also the source for some open questions. First, the derivation of the scaling law in the instantaneous momentum space formalism is rather simplistic and neglects both energy conservation and space-momentum correlations in the quark distributions. The observed scaling seems to put tight constraints on the latter [120, 121. The instantaneous formalism is also ill-prepared to distinguish between valence quark scaling in $p_{T}$ and $E_{T}$ because of the partial violation of energy and momentum conservation. Recent work using dynamical recombination with energy conservation 


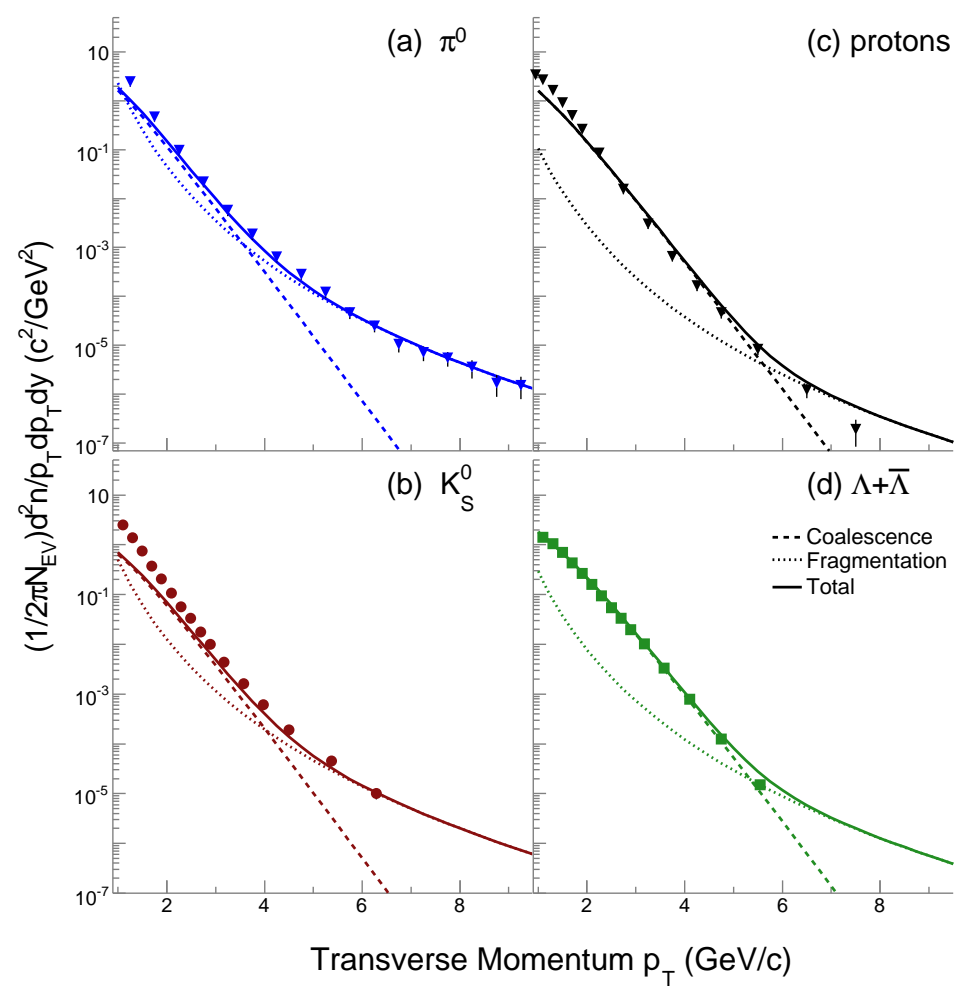

Figure 15: Hadron $p_{T}$-spectra at midrapidity from $200 \mathrm{GeV}$ central Au-Au collisions. The curves show the recombination and fragmentation components of the spectra obtained in the FMNB formalism along with the total which compares well with the data.

and realistic space-momentum correlations found that recombination respects scaling in $E_{T}$ [81. Future work in this direction is required to understand all aspects of the scaling phenomenon and its breaking [110, 112, 73, 122]. It will also be interesting to see how prominent recombination effects will be at the higher energies reached at the Large Hadron Collider LHC [123].

\section{Summary and conclusions}

In this chapter, we have presented two models of hadronization motivated by phenomenological observations in elementary and nuclear collisions.

The statistical model postulates that hadronization proceeds through the formation of massive colorless objects called clusters or fireballs, according to the general ideas of preconfinement of other cluster hadronization models [5]. They are formed in fragmentation in $\mathrm{e}^{+} \mathrm{e}^{-}$collisions just as they are in relativistic heavy ion collisions. While the size and charge distribution of these clusters differ between collision systems, each cluster gives rise to multihadronic states in a purely statistical fashion. Mathematically this can be described by a density matrix of localized hadronic states projected onto eigenstates 

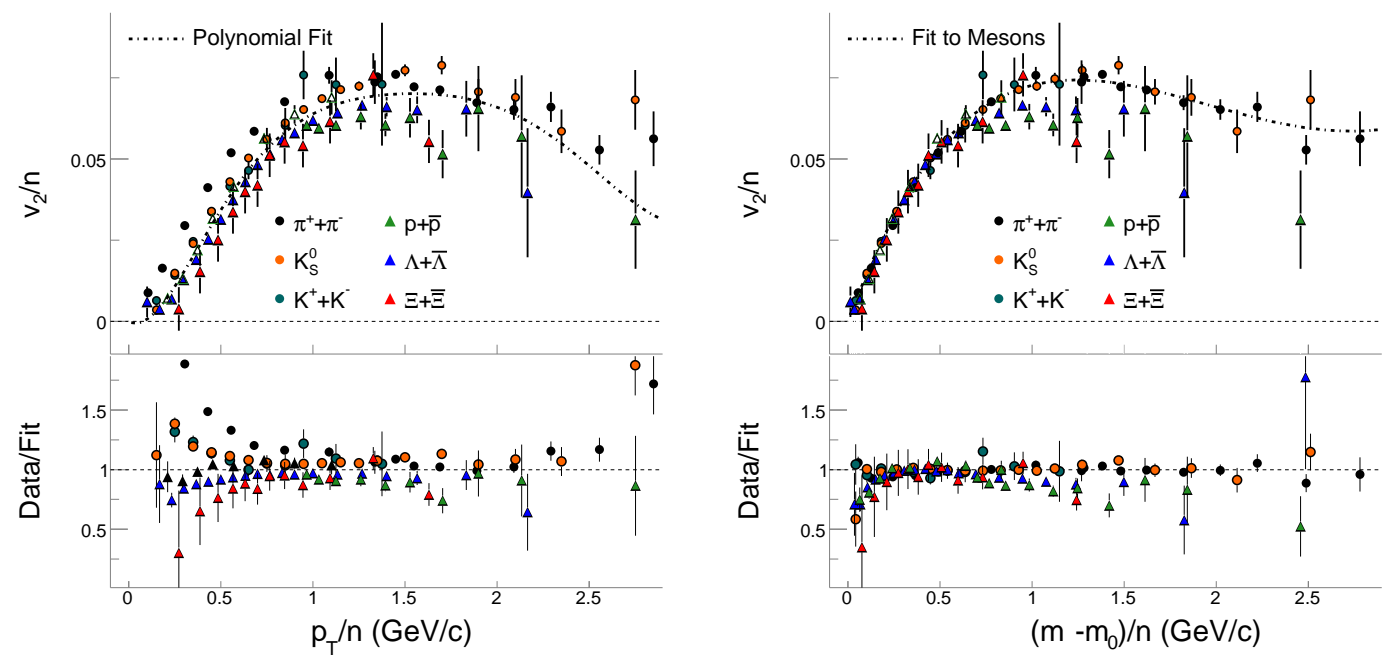

Figure 16: Top left panel: The elliptic anisotropy parameter $v_{2}$ scaled by quark number $n$ and plotted vs $p_{T} / n$. A polynomial curve is fit to all the data. The ratio of $v_{2} / n$ to the fit function is shown in the bottom left panel. Right panels: same but with $p_{T}$ replaced by $m_{T}-m_{0}$

with all possible hadronic quantum numbers. Interactions are, to a first approximation accommodated by including hadronic resonances (ideal hadron-resonance gas model).

The statistical model has been applied to a wide variety of small and large systems which differ by more than two orders of magnitude in the collision center of mass energy $\sqrt{s}$, revealing intriguing universal features of the hadronization process. The assumptions of the model were found to hold in a remarkable way for relative abundances and transverse momentum spectra of both light and heavy flavored species. For large enough ensembles of clusters a canonical statistical description can be used, which allows the introduction of a temperature $T$ of the clusters which is typically between 160 and 170 $\mathrm{MeV}$, corresponding to an average energy density of $\epsilon \approx 0.5 \mathrm{GeV} / \mathrm{fm}^{3}$ in a single cluster. An additional strangeness suppression parameter $\gamma_{s}$ is needed to reproduce the data, which is found to be between 0.5 and 0.7 in elementary collisions, while $\gamma_{s} \approx 1$ in central heavy ion collisions at RHIC. The origin of such an additional parameter and its relation to the strange quark mass are not clarified yet.

Clusters of hadrons at hadronization capture several important features of hadron production. It remains unspecified in this model how to connect it to the previous partonic phase in a microscopic picture. This step is complicated by the nature of the final partonic stage as an intermediate state in a large quantum system. This problem has been successfully resolved only for two simple cases: for single partons in high energy collisions described by vacuum fragmentation, and for the thermalized parton phase in heavy ion collisions which hadronizes through parton coalescence.

We have discussed the quark recombination model for heavy ion collisions and we have shown that this model recovers important features of the statistical model if applied 
to a thermalized parton phase. Experimental evidence for quark recombination comes from the Relativistic Heavy Ion Collider, in particular the spectacular valence quark scaling of elliptic flow. This is an important step to prove the existence of collective effects on the level of partons in heavy ion collisions [124]. While instantaneous recombination cannot capture the bulk of the hadronization process and is only applicable for momenta larger than 1 or $2 \mathrm{GeV} / c$, more recent models based on transport descriptions are able to address the urgent questions of energy conservation and entropy during hadronization. We have also shown a possible way to reconcile parton fragmentation with statistical hadronization via a recombination model for the full shower of partons created inside a jet cone.

Quark recombination and the statistical hadronization model offer a successful phenomenological description of many aspects of hadron production in elementary and nuclear collisions. They are based on few core principles which seem to capture key universal features in the process of hadron formation. While they do not answer the central questions of confinement and chiral symmetry breaking, which are of fundamental importance for QCD, they allow us to understand some of the key properties of QCD in the non-perturbative regime.

\section{Acknowledgements}

We are indebted to R. Stock for his encouragement and invaluable help while preparing this review. We thank P. Sorensen for his permission to use Figs. 12, 16.

\section{References}

[1] E. Fermi, Prog. Th. Phys. 5570 (1950).

[2] R. Hagedorn, Nuovo Cim. Suppl. 3147 (1965).

[3] J. Cleymans, H. Satz, Z. Phys. C 57135 (1993); P. Braun-Munzinger, J. Stachel, J. P. Wessels and N. Xu, Phys. Lett. B 3651 (1996); F. Becattini, M. Gazdzicki and J. Sollfrank, Eur. Phys. J. C 5143 (1998); P. Braun-Munzinger, D. Magestro, K. Redlich and J. Stachel, Phys. Lett. B 518, 41 (2001); A. Baran, W. Broniowski and W. Florkowski, Acta Phys. Polon. B 35779 (2004); J. Cleymans, B. Kampfer, M. Kaneta, S. Wheaton and N. Xu, Phys. Rev. C 71054901 (2005); F. Becattini, M. Gazdzicki, A. Keranen, J. Manninen and R. Stock, Phys. Rev. C 69024905 (2004);

[4] F. Becattini, Z. Phys. C 69485 (1996); F. Becattini and U. W. Heinz, Z. Phys. C 76269 (1997); F. Becattini and G. Passaleva, Eur. Phys. J. C 23551 (2002); F. Becattini, P. Castorina, J. Manninen and H. Satz, arXiv:0805.0964, to appear in Eur. Phys. J. C. 
[5] G. Corcella et al, J. High En. Phys., 0101:010 (2001) and references therein.

[6] D. Amati and G. Veneziano, Phys. Lett. B 83, 87 (1979).

[7] A. Chodos et al, Phys. Rev. D 93471

[8] F. Becattini and L. Ferroni, Eur. Phys. J. C 51, 899 (2007).

[9] F. Becattini and L. Ferroni, Eur. Phys. J. C 35, 243 (2004).

[10] F. Becattini, J. Phys. Conf. Ser. 5, 175 (2005).

[11] F. Becattini and L. Ferroni, Eur. Phys. J. C 52, 597 (2007).

[12] M. Chaichian, R. Hagedorn, M. Hayashi, Nucl. Phys. B 92445 (1975).

[13] S. Weinberg, The Quantum Theory Of Fields Vol. 1 Cambridge University Press.

[14] R. Dashen, S. Ma, H. Bernstein Phys. Rev. 187345 (1969);

R. Dashen, R. Rajamaran, Phys. Rev. D 10694 (1974).

[15] F. Becattini and L. Ferroni, Eur. Phys. J. C 38, 225 (2004).

[16] F. Becattini and G. Passaleva, Eur. Phys. J. C 23551 (2002).

[17] F. Becattini, P. Castorina, J. Manninen and H. Satz, arXiv:0805.0964, to appear in Eur. Phys. J. C.

[18] A. Keranen and F. Becattini, Phys. Rev. C 65, 044901 (2002).

[19] J. Rafelski and M. Danos, Phys. Lett. B 97, 279 (1980);

L. Turko, Phys. Lett. B 104, 153 (1981);

R. Hagedorn and K. Redlich, Z. Phys. C 27, 541 (1985);

S. Hamieh, K. Redlich and A. Tounsi, Phys. Lett. B 486, 61 (2000)

[20] U. W. Heinz, arXiv:hep-ph/0407360.

[21] F. Becattini, M. Gazdzicki, A. Keranen, J. Manninen and R. Stock, Phys. Rev. C 69, 024905 (2004).

[22] F. Becattini, M. Gazdzicki, J. Manninen, Phys. Rev. C 73, 044905 (2006).

[23] J. Manninen, F. Becattini, arXiv:0806.4100, Phys. Rev. C in press.

[24] J. Rafelski and B. Muller, Phys. Rev. Lett. 48, 1066 (1982).

[25] F. Becattini, M. Gazdzicki and J. Sollfrank, Eur. Phys. J. C 5 (1998) 143.

[26] J. Sollfrank, F. Becattini, K. Redlich and H. Satz, Nucl. Phys. A 638 (1998) 399C. 
[27] F. Antinori et al., NA57 coll., J. Phys. G 32, 427 (2006).

[28] B. I. Abelev et al., STAR Collaboration, Phys. Rev. C 77, 044908 (2008).

[29] S. Hamieh, K. Redlich and A. Tounsi, Phys. Lett. B 486 (2000) 61;

[30] J. Adams et al., STAR Collaboration, Phys. Rev. Lett. 98 (2007) 062301.

[31] J. Cleymans, B. Kampfer, P. Steinberg and S. Wheaton, Strangeness saturation: Energy and system-size dependence, arXiv:hep-ph/0212335; J. Cleymans, B. Kampfer, M. Kaneta, S. Wheaton and N. Xu, Phys. Rev. C 71054901 (2005).

[32] B. I. Abelev et al., STAR Collaboration, arXiv:0809.4737 [nucl-ex].

[33] F. Becattini and J. Manninen, J. Phys. G 35, 104013 (2008); F. Becattini and J. Manninen, arXiv:0811.3766 [nucl-th].

[34] C. Hohne, F. Puhlhofer and R. Stock, Phys. Lett. B 640, 96 (2006).

[35] P. Braun-Munzinger, J. Stachel and C. Wetterich, Phys. Lett. B 596, 61 (2004); C. Greiner, P. Koch-Steinheimer, F. M. Liu, I. A. Shovkovy and H. Stoecker, J. Phys. G 31, S725 (2005).

[36] U. Heinz and G. Kestin, PoS C POD2006, 038 (2006)

[37] R. Hagedorn, CERN lectures Thermodynamics of strong interactions (1970).

[38] F. Becattini and U. W. Heinz, Z. Phys. C 76269 (1997);

U. Heinz, Nucl. Phys. A 661140 (1999).

[39] R. Stock, Phys. Lett. B 456277 (1999).

[40] J. Hormuzdiar, S. D. H. Hsu, G. Mahlon, Int. J. Mod. Phys. E 12649 (2003).

[41] M. Srednicki, Phys. Rev. E 50888 (1994).

[42] M. Berry, J. Phys. A 102083 (1977).

[43] C. Jarzynski, Phys Rev E 56, 2254 (1997).

[44] A. Krzywicki, arXiv:hep-ph/0204116.

[45] T. S. Biro, S. G. Matinyan and B. Muller, Chaos and gauge field theory, World Sci. Lect. Notes Phys. 56, 1 (1994);

R. Pullirsch, K. Rabitsch, T. Wettig and H. Markum, Phys. Lett. B 427, 119 (1998).

[46] P. Castorina, D. Kharzeev and H. Satz, Eur. Phys. J. C 52, 187 (2007).

[47] J. F. Owens, Rev. Mod. Phys. 59, 465 (1987). 
[48] J. C. Collins, D. E. Soper and G. Sterman, Adv. Ser. Direct. High Energy Phys. 5, 1 (1988), Preprint arXiv:hep-ph/0409313.

[49] S. Albino, arXiv:0810.4255.

[50] J. C. Collins and D. E. Soper, Nucl. Phys. B 194, 445 (1982).

[51] B. A. Kniehl, G. Kramer and B. Potter, Nucl. Phys. B 582, 514 (2000);

[52] S. Albino, B. A. Kniehl and G. Kramer, Nucl. Phys. B 725, 181 (2005). S. Albino, B. A. Kniehl and G. Kramer, Nucl. Phys. B 803, 42 (2008).

[53] D. de Florian, R. Sassot and M. Stratmann, Phys. Rev. D 76, 074033 (2007).

[54] M. Adamovich et al. [WA82 Collaboration], Phys. Lett. B 305, 402 (1993).

[55] E. M. Aitala et al. [E791 Collaboration], Phys. Lett. B 371, 157 (1996).

[56] E. Braaten, Y. Jia and T. Mehen, Phys. Rev. Lett. 89, 122002 (2002).

[57] K. P. Das and R. C. Hwa, Phys. Lett. B 68, 459 (1977); Erratum-ibid. 73B, 504 (1978)].

[58] P. O. Bowman, U. M. Heller and A. G. Williams, Phys. Rev. D 66, 014505 (2002).

[59] J. I. Kapusta, Phys. Rev. C 21, 1301 (1980).

[60] C. Dover, U. Heinz, E. Schnedermann, and J. Zimányi, Phys. Rev. C 44, 1636 (1991); A. J. Baltz and C. Dover, Phys. Rev. C 53, 362 (1996); R. Mattiello et al., Phys. Rev. C 55, 1443 (1997).

[61] C. Gupt, R. K. Shivpuri, N. S. Verma and A. P. Sharma, Nuovo Cim. A 75, 408 (1983); J. A. Lopez, J. C. Parikh and P. J. Siemens, Phys. Rev. Lett. 53, 1216 (1984).

[62] T. S. Biro, P. Levai and J. Zimanyi, Phys. Lett. B 347, 6 (1995).

[63] T. S. Biro, P. Levai, and J. Zimanyi, J. Phys. G 28, 1561 (2002).

[64] J. Zimanyi, T. S. Biro, T. Csorgo and P. Levai, Phys. Lett. B 472, 243 (2000).

[65] L. Ravagli and R. Rapp, Phys. Lett. B 655, 126 (2007).

[66] V. Greco, C. M. Ko and P. Levai, Phys. Rev. Lett. 90, 202302 (2003);

[67] V. Greco, C. M. Ko and P. Levai, Phys. Rev. C 68, 034904 (2003).

[68] R. J. Fries, B. Muller, C. Nonaka and S. A. Bass, Phys. Rev. Lett. 90, 202303 (2003). 
[69] R. J. Fries, B. Muller, C. Nonaka and S. A. Bass, Phys. Rev. C 68, 044902 (2003).

[70] R. J. Fries, B. Muller, C. Nonaka and S. A. Bass, J. Phys. G 30, S223 (2004).

[71] R. C. Hwa and C. B. Yang, Phys. Rev. C 67, 034902 (2003).

[72] R. C. Hwa and C. B. Yang, Phys. Rev. C 70, 024905 (2004).

[73] B. Muller, R. J. Fries and S. A. Bass, Phys. Lett. B 618, 77 (2005).

[74] R. Rapp and E. V. Shuryak, Phys. Rev. D 67, 074036 (2003).

[75] R. J. Fries, J. Phys. G 30, S853 (2004).

[76] R. J. Fries, V. Greco and P. Sorensen, Ann. Rev. Nucl. Part. Sci. 58, 177 (2008).

[77] V. L. Chernyak and I. R. Zhitnitsky, Nucl. Phys. B 246, 52 (1984); S. J. Brodsky and G. P. Lepage, Adv. Ser. Direct. High Energy Phys. 5, 93 (1989).

[78] E. M. Aitala et al. [E791 Collaboration], Phys. Rev. Lett. 86, 4768 (2001).

[79] A. P. Bakulev, S. V. Mikhailov and N. G. Stefanis, Phys. Lett. B 508, 279 (2001); Erratum-ibid. B 590, 309 (2004).

[80] J. Zimanyi, P. Levai and T. S. Biro, J. Phys. G 31, 711 (2005).

[81] L. Ravagli, H. van Hees and R. Rapp, arXiv:0806.2055 [hep-ph].

[82] S. Afanasiev et al. [PHENIX Collaboration], Phys. Rev. Lett. 99, 052301 (2007).

[83] V. Greco, Eur. Phys. J. Special Topics 155, 45 (2008).

[84] C. Nonaka, B. Muller, M. Asakawa, S. A. Bass and R. J. Fries, Phys. Rev. C 69, 031902 (2004).

[85] R. C. Hwa and C. B. Yang, Phys. Rev. C 70, 024904 (2004).

[86] R. C. Hwa and C. B. Yang, Phys. Rev. Lett. 93, 082302 (2004); R. C. Hwa, C. B. Yang and R. J. Fries, Phys. Rev. C 71, 024902 (2005).

[87] R. C. Hwa, C. B. Yang and R. J. Fries, Phys. Rev. C 71, 024902 (2005).

[88] R. C. Hwa and C. B. Yang, Phys. Rev. C 70, 054902 (2004).

[89] R. J. Fries, S. A. Bass and B. Muller, Phys. Rev. Lett. 94, 122301 (2005).

[90] R. J. Fries, J. Phys. G 31, S379 (2005).

[91] R. J. Fries, J. Phys. Conf. Ser. 27, 70 (2005).

[92] A. Majumder, E. Wang and X. N. Wang, Phys. Rev. C 73, 044901 (2006). 
[93] S. S. Adler et al. [PHENIX Collaboration], Phys. Rev. Lett. 91, 172301 (2003).

[94] J. Adams et al. [STAR Collaborations], preprint arXiv:nucl-ex/0601042; H. Long [STAR Collaboration], J. Phys. G 30, S193 (2004).

[95] J. Adams et al. [STAR Collaboration], Phys. Rev. Lett. 92, 052302 (2004).

[96] J. Adams et al. [STAR Collaboration], Phys. Rev. C 72, 014904 (2005).

[97] S. S. Adler et al. [PHENIX Collaboration], Phys. Rev. Lett. 91, 182301 (2003).

[98] J. Adams et al. [STAR Collaboration], Phys. Rev. Lett. 95, 122301 (2005).

[99] P. Abreu et al. [DELPHI Collaboration], Eur. Phys. J. C 17, 207 (2000).

[100] B. Alper et al. [British-Scandinavian Collaboration], Nucl. Phys. B 100, 237 (1975).

[101] V. Greco and C. M. Ko, J. Phys. G 31 (2005) S407.

[102] C. Nonaka, R. J. Fries and S. A. Bass, Phys. Lett. B 583, 73 (2004).

[103] R. Baier, D. Schiff and B. G. Zakharov, Ann. Rev. Nucl. Part. Sci. 50, 37 (2000); M. Gyulassy, I. Vitev, X. N. Wang and B. W. Zhang, preprint arXiv:nucl-th/0302077; Jet quenching is discussed in more details in other chapters of this volume. note: reference

[104] J. Adams et al. [STAR Collaboration], Phys. Rev. Lett. 98, 062301 (2007).

[105] J. Adams et al. [STAR Collaboration], Phys. Rev. C 71, 064902 (2005).

[106] B. I. Abelev et al. [STAR Collaboration], Phys. Rev. Lett. 99, 112301 (2007).

[107] B. I. Abelev et al. [STAR Collaboration], Phys. Rev. Lett. 97, 152301 (2006).

[108] S. Voloshin and Y. Zhang, Z. Phys. C 70, 665 (1996); A. M. Poskanzer and S. A. Voloshin, Phys. Rev. C 58, 1671 (1998).

[109] S. A. Voloshin, Nucl. Phys. A 715, 379 (2003).

[110] V. Greco and C. M. Ko, preprint $\operatorname{arXiv:nucl-th/0505061.~}$

[111] D. Molnar and S. A. Voloshin, Phys. Rev. Lett. 91, 092301 (2003);

[112] Z. w. Lin and D. Molnar, Phys. Rev. C 68, 044901 (2003).

[113] V. Greco, C. M. Ko, and R. Rapp, Phys. Lett. B 595, 202 (2004).

[114] P. F. Kolb, L. W. Chen, V. Greco and C. M. Ko, Phys. Rev. C 69, 051901 (2004). Eur. Phys. J. Special Topics 155, 45 (2008). 
[115] B. I. Abelev et al. [STAR Collaboration], preprint arXiv:0801.3466 [nucl-ex].

[116] P. Huovinen, P. F. Kolb, U. W. Heinz, P. V. Ruuskanen and S. A. Voloshin, Phys. Lett. B 503, 58 (2001), P. F. Kolb, AIP Conf. Proc. 698, 694 (2004).

[117] P. F. Kolb and U. W. Heinz, in R. C. Hwa and X. N. Wang (ed.), Quark Gluon Plasma 3, World Scientific, Singapore, 634 (2004), preprint arXiv:nucl-th/0305084.

[118] T. Hirano and Y. Nara, Phys. Rev. C 69, 034908 (2004).

[119] C. Adler et al. [STAR Collaboration], Phys. Rev. Lett. 89, 132301 (2002).

[120] S. Pratt and S. Pal, Nucl. Phys. A 749, 268 (2005).

[121] D. Molnar, preprint arXiv:nucl-th/0408044.

[122] V. Greco and C. M. Ko, Phys. Rev. C 70, 024901 (2004).

[123] R. J. Fries and B. Muller, Eur. Phys. J. C 34, S279 (2004).

[124] P. Sorensen, J. Phys. G 32, S135 (2006). 